\title{
Modulated spin-liquid and magnetic order from a Kondo-Heisenberg model applied to $\mathrm{URu}_{2} \mathrm{Si}_{2}$
}

\author{
X. Montiel* \\ International Institute of Physics, Universidade Federal do Rio Grande do Norte, 59078-400 Natal-RN, Brazil \\ S. Burdin ${ }^{\dagger}$ \\ Univ. Bordeaux, LOMA, UMR 5798, F-33400 Talence, France and CNRS, LOMA, UMR 5798, F-33400 Talence, France \\ C. Pépin \\ Institut de Physique Théorique, CEA-Saclay, 91191 Gif-sur-Yvette, France
}

\author{
A. Ferraz \\ International Institute of Physics, Universidade Federal do Rio Grande do Norte, 59078-400 Natal-RN, Brazil \\ and Departamento de Física Teórica e Experimental, Universidade Federal do Rio Grande do Norte, 59072-970 Natal-RN, Brazil
}

(Received 11 December 2013; revised manuscript received 4 June 2014; published 22 July 2014)

\begin{abstract}
Using the Kondo-Heisenberg model framework, we analyze the effect of charge fluctuations in the modulated spin liquid (MSL) and in the antiferromagnetic (AF) ordering which were introduced by Pépin et al. [Phys. Rev. Lett. 106, 106601 (2011)] and Thomas et al. [Phys. Rev. B 87, 014422 (2013)]. Coupling the spin liquid to the charge sector enables us to discuss the formation of the Kondo effect in this system. As a result, we are able to observe the emergence of two phases showing the coexistence of the Kondo effect and the MSL (Kondo-MSL phase) and the coexistence of the Kondo-MSL-AF phases. We discuss the critical lines separating the coexisting Kondo-MSL and Kondo-MSL-AF phases. Our model produces promising results as regards to the experimental temperature-pressure phase diagram and to the two gap openings observed in $\mathrm{URu}_{2} \mathrm{Si}_{2}$.
\end{abstract}

DOI: 10.1103/PhysRevB.90.045125

\section{INTRODUCTION}

Kondo lattices are among the most studied models in condensed matter physics [1]. They are realized in broad families of strongly correlated materials, when conduction electrons interact with the periodic crystal of quantum magnetic moments. In heavy-fermion compounds, the local moments represent the $f$ electrons from the lanthanide or actinide atoms. An extremely rich variety of unusual quantum phases is observed in those systems [2-4]. Exotic superconductivity [5], quantum critical points (QCP) [6,7], and their related nonstandard critical exponents result from the competition between the various microscopic mechanisms [8-11].

$\mathrm{URu}_{2} \mathrm{Si}_{2}$ is one of the most mysterious and fascinating compounds in the field of condensed matter. A second-order phase transition below $17.5 \mathrm{~K}$ was discovered in 1986 through very clear thermodynamic and transport signatures [12-14]. As a marker of that, the entropy loss per $U$ atoms at the transition is approximately $0.3 k_{B} \ln 2$ [12]. Although magnetism is a natural candidate to induce most phase transitions in heavy metals, it was promptly observed by inelastic neutron scattering (INS) experiments [15,16] that even if this phase transition were of magnetic origin, the magnetic moments involved would be extremely small in magnitude [15]. This observation invalidated the formation of a spin density wave (SDW) as a candidate for the "hidden order" (HO) in $\mathrm{URu}_{2} \mathrm{Si}_{2}$. The response in the charge sector was tested in earlier days via $\mathrm{X}$-ray diffraction [17]. The symmetry of the order parameter

\footnotetext{
*x.montiel1@gmail.com

†sébastien.burdin@u-bordeaux1.fr
}

PACS number(s): 72.80.Ga, 74.40.Kb, 75.10.Kt, 75.25.Dk seemed to resist characterization through standard diffraction techniques [18].

Recently, new experimental developments have revived this field. A more complete set of INS experiments [19-22] has drawn a very striking parallel between the $\mathrm{HO}$ and the antiferromagnetic (AFM) phases obtained under pressure. In particular, the temperature-pressure (T-P) phase diagram shows that, under pressure, the compound undergoes a secondorder phase transition at a Néel temperature, towards an AFM phase, of the same magnitude as that of the $\mathrm{HO}$ transition. A first-order transition is observed between the $\mathrm{HO}$ and AFM when pressure is increased at constant T. Very strikingly, no sign of Fermi surface reconfiguration is observed while the resistivity changes continuously [23] and the magnetic oscillations experiment shows no shift of the band frequencies at that HO-AFM transition [24]. It can be deduced from this observation that the HO phase displays the same doubling of the Brillouin zone (BZ) of the AFM phase. From the magnetic oscillation measurements [24], a very strong Ising-type anisotropy can be inferred in both AFM and $\mathrm{HO}$ phases. Besides, scanning tunneling microscopy experiments (STM) [25] report the opening of a hybridization gap in the electronic spectrum. Studies by point contact spectroscopies [26] and angle-resolved photoemission spectroscopy (ARPES) experiments [27,28] promote the picture of the opening of a hybridization gap at a higher temperature $(\sim 70 \mathrm{~K})$ than that of the HO phase transition. The question of whether the opening of the hybridization gap is influential for the formation of the $\mathrm{HO}$ is an important issue which is still unresolved at the present time [29-32].

During the last 30 years, several theories have been proposed to explain the HO phase. The localized theories rely on the complexity of the $\mathrm{U}$ orbitals to stabilize a multipolar 
orbital order in that compound. These models lead to a site-localized order parameter. Increasing step by step the complexity of the selected order, these approaches started up with a quadrupolar order parameter [33], then successively proposed octopolar or hexadecapolar orderings [34-36], the most complex order being so far the dotriacontapolar order parameter [37]. On the other hand, several authors proposed an itinerant order parameter in nature and then came out with alternative scenarios based on the condensation of waves of itinerant electrons with various symmetry breakings [38-43].

However, the study of $U$ atoms based compounds reveal the dual localized-itinerant character of the U atoms' $5 f$ electrons (for example, in $\mathrm{UPd}_{2} \mathrm{Al}_{3}$ [44]). This gives rise to dual model in which the $5 f$ electrons are both localized and itinerant (see, for example, the model developed for $\mathrm{UPt}_{3}$ in Ref. [45]).

This dual aspect of $5 f$ electrons in $\mathrm{URu}_{2} \mathrm{Si}_{2}$ is well accepted (Ref. [18]). Yet, the initial cleavage between localized versus itinerant order parameter evolved to model with a site-localized order parameter (multipolar model [33-37] or hastatic model [46]) versus model with a bond order parameter $[47,49,50]$. Note that a classification of the symmetries compatible with the hidden order phase has been provided in Ref. [48].

Recently, we proposed a theory in which the HO phase is identified with a modulated spin liquid (MSL) [49,50] with some resonant valence bonds forming a sort of singlet crystal. A similar order parameter, which is also defined on the dual lattice space, has been proposed by Riseborough et al. in Ref. [47].

Our approach is based on the idea that the hidden order is characterized by a many-body wave function in which magnetic degrees of freedom are highly entangled from site to site. The commensurate wave vector emerges naturally from this intersite entanglement as discussed elsewhere [49,50]. The lattice translation symmetry of the MSL state is broken in the same way as in the AFM phase, but the point-group symmetries of the MSL and AFM states may be different from each other. Note that the point-group symmetry analysis of Harima [48] should provide strong constraints in the selection of the appropriate MSL order parameter.

Up to now, we have developed the MSL concept within a localized picture, both in the case of the quantum Heisenberg model on the square lattice [49] and in the more realistic tetragonal body-centered structure in three dimensions (3D), specific of this compound [50]. The calculations of the MSL model performed on the realistic 3D structure [50] confirm the results initially obtained on two-dimensional (2D) square lattice [49]. Furthermore, a deeper study shows that frustration could emerge from the 3D structure as discussed in Ref. [51]. The MSL has no local magnetization but it breaks the lattice translation symmetry with the same commensurate wave vector as in the AFM order. Through the AF to MSL phase transition, the staggered magnetization vanishes, restoring the time-reversal symmetry. At the same time, the MSL order parameter rises preserving the lattice symmetry breaking. In this MSL scenario, the partial melting of the AF order explains most of the physical properties of the AF to $\mathrm{HO}$ transition in $\mathrm{URu}_{2} \mathrm{Si}_{2}$. Yet, this material is also a metallic heavy fermion and the Kondo effect also needs to be taken into account for a correct description of its physical properties.
The aim of this paper is to study the stability of the MSL with respect to a coupling to the charge sector via the hybridization to a light conduction band. These considerations led us to analyze Fermi surfaces for the Kondo lattice model with an extra explicit Ruderman-Kittel-Kasuya-Yosida (RKKY) interaction that is able to reproduce the MSL-AF transition. A complex phase diagram emerges out of that in which we can observe the formation of Kondo states and discuss the stability of the MSL and AFM phases as well.

Considering the complexity of this task, we decided to focus again on the square lattice in this preliminary investigation and leave the more realistic 3D lattice and multiorbital cases for a later study.

In this paper, we neglect the orbital character of the $5 f$ electrons of the $\mathrm{U}$ atoms. However, the dual aspect of the $5 f$ electrons of $\mathrm{U}$ atoms allows us to model them as having both a localized and an itinerant component (see for example Ref. [45]). This decomposition leads to a type of Kondo system (studied for example in [43]) where a RKKY interaction can exist [52]. Reducing the realistic system to a Kondo lattice (where $5 f$ electrons are decoupled in a local moment component and in a conduction electron component), we lose the local configuration information but this approximation allows us to study the bond order parameter. We also assume the existence of frustration in the system and we phenomenologically consider a spin-liquid order parameter in the system. The study of the frustration in bct system is considered in Ref. [51].

This paper is organized as follows: In Sec. II, we present the general model and the method used to establish the phase diagram presented in Sec. III. In Sec. IV, we apply this general framework to the particular case of $\mathrm{URu}_{2} \mathrm{Si}_{2}$. The analyses of the band structure and of the Fermi surface, with emphasis on the characteristic signatures of each order parameter, are presented in the Appendices.

\section{GENERAL MODEL AND METHOD}

\section{A. Model}

We consider a Kondo-Heisenberg model $[53,54]$ on a square lattice with $N$ sites and a lattice constant $a=1$, defined by the following Hamiltonian:

$$
\begin{aligned}
H & =H_{\mathrm{KL}}+H_{\mathrm{RKKY}} \\
& \equiv t_{c} \sum_{\left\langle R, R^{\prime}\right\rangle, \alpha} c_{R \alpha}^{\dagger} c_{R^{\prime} \alpha}+J_{K} \sum_{R} \mathbf{S}_{R} \cdot \mathbf{s}_{R}+J \sum_{\left\langle R, R^{\prime}\right\rangle} \mathbf{S}_{R} \cdot \mathbf{S}_{R^{\prime}},
\end{aligned}
$$

where the operator $c_{R \alpha}^{(\dagger)}$ annihilates (creates) a conduction electron on site $R$ with spin component $\alpha=\uparrow, \downarrow \equiv \pm 1$, and $\mathbf{S}_{R}$ denotes a quantum spin $\frac{1}{2}$ on site $R$. The sum over $\left\langle R, R^{\prime}\right\rangle$ refers to nearest neighbors with each bond being counted only once. The conduction electron local spin density can be expressed as $\mathbf{s}_{R}=\frac{1}{2} \sum_{\alpha \beta} c_{R \alpha}^{\dagger} \sigma_{\alpha \beta} c_{R \beta}$, where $\sigma \equiv\left(\sigma^{x}, \sigma^{y}, \sigma^{z}\right)$ denotes the Pauli matrices. The average electronic occupation per site is fixed to be $n_{c}$, which will later be taken into account by the introduction of a given chemical potential $\mu$. Here, $H_{\mathrm{KL}}$ is a Kondo lattice Hamiltonian, with a nearest-neighbor 
hopping term $t_{c}$ and a local Kondo antiferromagnetic coupling $J_{K}$ between conduction electrons and local moments. The Heisenberg term $H_{\mathrm{RKKY}}$ adds an antiferromagnetic interaction $J$ between nearest-neighboring Kondo spins.

Hereafter, we use the Abrikosov pseudofermions representation for the local spin- $\frac{1}{2}$ operators [55]: $\mathbf{S}_{R}=$ $\frac{1}{2} \sum_{\alpha \beta} f_{R \alpha}^{\dagger} \sigma_{\alpha \beta} f_{R \beta}$, where the $f_{R \alpha}\left(f_{R \alpha}^{\dagger}\right)$ are fermionic annihilation (creation) operators satisfying the local single-particle occupation constraint

$$
f_{R \uparrow}^{\dagger} f_{R \uparrow}+f_{R \downarrow}^{\dagger} f_{R \downarrow}=1 .
$$

The Kondo interaction is first rewritten as $J_{K} \mathbf{S}_{R} \cdot \mathbf{s}_{R}=$ $\frac{J_{K}}{2}\left(\sum_{\alpha} f_{R \alpha}^{\dagger} c_{R \alpha}\right)\left(\sum_{\beta} f_{R \beta} c_{R \beta}^{\dagger}\right)-J_{K} n_{c} / 4$. These local terms are then decoupled using a standard mean-field approximation [56-58], within the Hubbard-Stratonovitch scheme. The RKKY interaction is treated with exactly the same mean-field procedure as the one described in Ref. [49]: the Heisenberg interaction is decoupled on each nearest-neighbor bond $\left\langle R, R^{\prime}\right\rangle$, partially in a spin-liquid channel, and partially in an antiferromagnetic Weiss field channel. The respective weights $J_{\mathrm{SL}} / J$ and $J_{\mathrm{AF}} / J$ of each decoupling channel are constrained by the relation $J \equiv J_{\mathrm{SL}}+J_{\mathrm{AF}}$. Here, $J_{\mathrm{SL}}$ and $J_{\mathrm{AF}}$ can be considered as tuning parameters for the model. This decoupling scheme, which might appear to be arbitrary on a square lattice model, captures phenomenologically some of the frustration effects of a more realistic three-dimensional model [50,51].

\section{B. Mean-field method}

We introduce the mean-field decouplings in the Kondo, the Weiss-AF, and the SL channels, considering only the collinear order for the AF channel. The Hamiltonian (1) is approximated as

$$
\begin{aligned}
H \approx & H_{\mathrm{MF}} \equiv \sum_{\alpha}\left(H_{\mathrm{KL}}^{\alpha}+H_{\mathrm{RKKY}}^{\alpha}\right)+E_{0} \\
& +\mu \sum_{R, \alpha}\left(\frac{n_{c}}{2}-c_{R \alpha}^{\dagger} c_{R \alpha}\right)+\lambda \sum_{R, \alpha}\left(\frac{1}{2}-f_{R \alpha}^{\dagger} f_{R \alpha}\right),
\end{aligned}
$$

with

$$
\begin{aligned}
H_{\mathrm{KL}}^{\alpha}= & t_{c} \sum_{\left\langle R, R^{\prime}\right\rangle} c_{R \alpha}^{\dagger} c_{R^{\prime} \alpha}+\sum_{R}\left(b_{R} c_{R \alpha}^{\dagger} f_{R \alpha}+\text { H.c. }\right), \\
H_{\mathrm{RKKY}}^{\alpha}= & \sum_{\left\langle R, R^{\prime}\right\rangle}\left(\varphi_{R R^{\prime}} f_{R \alpha}^{\dagger} f_{R^{\prime} \alpha}+\text { H.c. }\right) \\
& +\alpha \sum_{R} f_{R \alpha}^{\dagger} f_{R \alpha} \sum_{z} m_{R+z}, \\
E_{0}= & \sum_{R} \frac{\left|b_{R}\right|^{2}}{J_{K}}+\sum_{\left\langle R, R^{\prime}\right\rangle}\left(\frac{\left|\varphi_{R R^{\prime}}\right|^{2}}{J_{\mathrm{SL}}}-\frac{m_{R} m_{R^{\prime}}}{2 J_{\mathrm{AF}}}\right),
\end{aligned}
$$

where $\lambda$ is a Lagrange multiplier introduced in order to take into account the local constraints (2). Following the standard mean-field scheme [56-58], this Lagrange multiplier field has been assumed to be constant and homogeneous. Therefore, $\lambda$ acts here as an effective chemical potential for the Abrikosov fermions and the constraints (2) are satisfied on average only. The sum over $z$ denotes a sum over all the nearestneighbor sites. The Kondo mean-field parameter is given by the self-consistent equation $b_{R}=J_{K} \sum_{\alpha}\left\langle f_{R \alpha}^{\dagger} c_{R \alpha}\right\rangle$, where the thermal average $\langle\ldots\rangle$ is computed from the mean-field Hamiltonian (3). This Kondo parameter can be understood as an effective hybridization between the light conduction electron band and the heavy flat band [56-59]. The spin-liquid and antiferromagnetic mean-field self-consistent relations give $\varphi_{R R^{\prime}}=-J_{\mathrm{SL}} \sum_{\alpha}\left\langle f_{R \alpha}^{\dagger} f_{R^{\prime} \alpha}\right\rangle$ and $m_{R}=J_{\mathrm{AF}} \sum_{\alpha}\left\langle\alpha f_{R \alpha}^{\dagger} f_{R \alpha}\right\rangle$. A full self-consistent resolution of the mean-field effective model also requires the determination of $\mu$ and $\lambda$ from the relations $\frac{1}{N} \sum_{R, \alpha}\left\langle c_{R \alpha}^{\dagger} c_{R \alpha}\right\rangle=n_{c}$ and $\frac{1}{N} \sum_{R, \alpha}\left\langle f_{R \alpha}^{\dagger} f_{R \alpha}\right\rangle=1$. Note that a similar bond order parameter was also proposed in Ref. [47] in the framework of an underscreened Anderson model.

In principle, the Kondo and the Weiss fields $b_{R}$ and $m_{R}$ are site dependent whereas the SL field $\varphi_{R R^{\prime}}$ is bond dependent. Following the procedure of Refs. [49,50], we will make some simplifying ansatz for the RKKY fields.

First, we notice that the AF field $m_{R}$ is formally similar to the standard Weiss mean-field moment that would emerge from a classical approximation. Since we consider a square lattice model, we will consider only the Néel ordering to represent the AF state. We thus assume that $m_{R}=S_{Q} e^{i Q R}$, where $Q=$ $(\pi, \pi)$ and $S_{Q}$ is the staggered magnetization.

The SL field $\varphi_{R R^{\prime}}$ is reminiscent of the resonant valence bond (RVB) state introduced by Anderson and coworkers $[60,61]$. The RVB state was originally introduced within a homogeneous SL field. This was motivated by the description of frustrated spin systems that could not form a long-range magnetically ordered state. Nevertheless, it was shown in Ref. [49] that a spatially modulated spin liquid (MSL) was more energetically stable on a square lattice than a homogeneous SL phase. Here, we assume a real-space modulation of the SL mean field which has the form $\varphi_{R R^{\prime}}=$ $\delta_{R R^{\prime}} \phi_{0}+i \frac{\phi_{Q}}{2} \sum_{ \pm} e^{ \pm i Q\left(R+R^{\prime}\right) / 2}$. The SL field is defined on the dual (i.e., bond) lattice in general, although this particular MSL ansatz breaks the same lattice translation symmetry which is broken by the AF Néel ordering. Consequently, the Bravais lattices for the MSL and AF ordered state are identical to each other and correspond to a doubled unit cell in contrast with the single unit of the initial square lattice.

Here, we make another ansatz for the Kondo parameter $b_{R}$. An indirect coupling between the modulated spin-liquid order parameter and the Kondo screening may produce a modulation of the Kondo parameter as well. Thus, we include the possibility of a modulation for the Kondo mean-field parameter writing $b_{R}=b_{0}+i b_{Q} e^{i Q R}$, where both $b_{0}$ and $b_{Q}$ are real. With this ansatz, the phase of the Kondo mean-field parameter can be spatially modulated, when $b_{Q} \neq 0$. But, its amplitude $\rho \equiv \sqrt{b_{0}^{2}+b_{Q}^{2}}$ is homogeneous since $e^{i Q R}$ reduces only to \pm 1 . Note that the amplitude $\rho$ could also be modulated. Indeed, a space modulation of $\rho$ may lead to charge ordering. We left this possibility aside because it has never been observed in the $\mathrm{URu}_{2} \mathrm{Si}_{2}$ compound which is the main motivation of this work. Finally, the Kondo parameters are restated as $b_{0}=\rho \cos (\theta)$ and $b_{Q}=\rho \sin (\theta)$, with a nonzero value of the amplitude $\rho$, typical for a Kondo phase. A nonzero angle $\theta$ clearly indicates that the Kondo phase is modulated. We define the Kondo temperature $T_{K}$ as the temperature at which 
the conduction electrons start to screen the local moments. Numerically, we signaled this temperature as the temperature where the amplitude $\rho$ becomes different from zero.

Invoking the Fourier transform $f_{k \alpha} \equiv \frac{1}{\sqrt{N}} \sum_{R} e^{i k R} f_{R \alpha}$ and its inverse, and replacing the site- and bond-dependent mean fields by their corresponding ansatz expressions, the effective mean-field Hamiltonian (3) reads as

$$
\begin{aligned}
H_{\mathrm{MF}}= & \sum_{k, \alpha}\left[\left(t_{c} \gamma_{k}-\mu\right) c_{k \alpha}^{\dagger} c_{k \alpha}+\left(\phi_{0} \gamma_{k}-\lambda\right) f_{k \alpha}^{\dagger} f_{k \alpha}\right] \\
& +\sum_{k, \alpha}\left(4 \alpha S_{Q}+i \phi_{Q} \gamma_{k-Q / 2}\right) f_{k \alpha}^{\dagger} f_{k-Q \alpha} \\
& +\rho \cos (\theta) \sum_{k, \alpha}\left(c_{k \alpha}^{\dagger} f_{k \alpha}+f_{k \alpha}^{\dagger} c_{k \alpha}\right) \\
& +i \rho \sin (\theta) \sum_{k, \alpha}\left(f_{k-Q \alpha}^{\dagger} c_{k \alpha}-c_{k-Q \alpha}^{\dagger} f_{k \alpha}\right) \\
& +N\left[\frac{\rho^{2}}{J_{K}}+\frac{2\left(\phi_{0}^{2}+\phi_{Q}^{2}\right)}{J_{\mathrm{SL}}}+\frac{S_{Q}^{2}}{J_{\mathrm{AF}}}+\lambda+\mu n_{c}\right]
\end{aligned}
$$

where $\gamma_{k} \equiv-2 \cos \left(k_{x}\right)-2 \cos \left(k_{y}\right)$ is the square lattice dispersion.

The mean-field parameters $\phi_{0}, \phi_{Q}, S_{Q}, \rho, \theta$, the Lagrange multiplier $\lambda$, and the chemical potential $\mu$ are determined selfconsistently by the minimization of the free energy, given by $\beta \mathcal{F}_{\mathrm{MF}}=-\operatorname{Tr}\left[\exp \left(-\beta H_{\mathrm{MF}}\right)\right]$. We find the following saddlepoint equations:

$$
\begin{gathered}
\phi_{0}=-\frac{J_{\mathrm{SL}}}{4 N} \sum_{k, \alpha} \gamma_{k}\left\langle f_{k \alpha}^{\dagger} f_{k \alpha}\right\rangle, \\
\phi_{Q}=-\frac{i J_{\mathrm{SL}}}{4 N} \sum_{k, \alpha} \gamma_{k-Q / 2}\left\langle f_{k \alpha}^{\dagger} f_{k-Q \alpha}\right\rangle,
\end{gathered}
$$

$$
\begin{gathered}
S_{Q}=-\frac{2 J_{\mathrm{AF}}}{N} \sum_{k, \alpha} \alpha\left\langle f_{k \alpha}^{\dagger} f_{k-Q \alpha}\right\rangle, \\
\rho \cos (\theta)=-\frac{J_{K}}{2 N} \sum_{k, \alpha}\left\langle c_{k \alpha}^{\dagger} f_{k \alpha}+\text { H.c. }\right\rangle, \\
\rho \sin (\theta)=-\frac{J_{K}}{2 N} \sum_{k, \alpha}\left\langle i f_{k-Q \alpha}^{\dagger} c_{k \alpha}+\text { H.c. }\right\rangle, \\
1=\frac{1}{N} \sum_{k, \alpha}\left\langle f_{k \alpha}^{\dagger} f_{k \alpha}\right\rangle, \\
n_{c}=\frac{1}{N} \sum_{k, \alpha}\left\langle c_{k \alpha}^{\dagger} c_{k \alpha}\right\rangle .
\end{gathered}
$$

\section{Numerical treatment of the mean-field equations}

Within the mean-field Hamiltonian, the free energy per site can be written explicitly as a function of the mean-field parameters $\phi_{0}, \phi_{Q}, S_{Q}, \rho, \theta$, the Lagrange multiplier $\lambda$, and the chemical potential $\mu$. We find

$$
\begin{aligned}
\mathcal{F}_{\mathrm{MF}}= & -\frac{k_{B} T}{N} \sum_{k, n} \ln \left(1+e^{\left(-\beta \Omega_{n}\right)}\right)+\lambda+\mu n_{c} \\
& +\frac{\rho^{2}}{J_{K}}+\frac{2\left(\phi_{0}^{2}+\phi_{Q}^{2}\right)}{J_{\mathrm{SL}}}+\frac{S_{Q}^{2}}{J_{\mathrm{AF}}},
\end{aligned}
$$

where $\beta=1 / k_{B} T, N$ is the number of sites, $n$ runs over the band defined by the eigenvalues of the effective meanfield Hamiltonian, and $\Omega_{n}$ are the eigenvalues of the following mean-field Hamiltonian matrix

$$
\left(\begin{array}{cccc}
t_{c} \gamma_{k}-\mu & 0 & \rho \cos (\theta) & -i \rho \sin (\theta) \\
0 & t_{c} \gamma_{k-Q}-\mu & -i \rho \sin (\theta) & \rho \cos (\theta) \\
\rho \cos (\theta) & i \rho \sin (\theta) & \phi_{0} \gamma_{k}-\lambda & 4 \alpha S_{Q}+i \phi_{Q} \gamma_{k-\frac{Q}{2}} \\
i \rho \sin (\theta) & \rho \cos (\theta) & 4 \alpha S_{Q}-i \phi_{Q} \gamma_{k-\frac{Q}{2}} & \phi_{0} \gamma_{k-Q}-\lambda
\end{array}\right)
$$

For a numerical self-consistent solution, we proceed iteratively as follows. Within each loop, for a fixed $\left(\phi_{0}, \phi_{Q}, S_{Q}, r_{0}, r_{Q}\right)$, the chemical potentials $\lambda$ and $\mu$ are determined by dichotomy method in order to fulfill simultaneously the constraint conditions (11) or (12). In turn, for both fixed $\lambda$ and $\mu$, the free-energy expression (13) is minimized using Powells method [62], providing the solution $\left(\phi_{0}, \phi_{Q}, S_{Q}, \rho, \theta\right)$ of the mean-field equations (6)-(10).

All the numerical results presented in this paper have been obtained with the following choice of parameters: the Kondo coupling is set to be $J_{K} / t_{c}=0.8686$ where $t_{c}$ is the conduction electron hopping. $t_{c}$ characterizes the conduction electron bandwidth which equals $8 t_{c}$ for a square lattice. These parameters are chosen so that the Kondo temperature $T_{K 0}$ for a zero RKKY interaction $\left(J_{\mathrm{AF}}=J_{\mathrm{SL}}=0\right)$ is small compared to the bandwidth $\left(T_{K 0}=0.098 t_{c}\right)$. This guarantees that we work in the weak Kondo coupling regime $T_{K} \ll t_{c}$. We also choose an electronic filling $n_{c}=0.7$ which avoids both the square lattice instability (at $n_{c}=0.5$ ) and the Kondo insulating regime (at $n_{c}=1$ ). We used other sets of numerical values for testing, that are not presented here, and we obtained the same qualitative conclusions as the ones presented in this paper.

\section{PHASE DIAGRAM}

In this section, we analyze the phase diagram obtained for the Kondo-Heisenberg model within our mean-field approximation. Considering the different mean-field parameters introduced in the previous section, $\phi_{0}, \phi_{Q}, S_{Q}, \rho$, and $\theta$, we study the competition or the cooperation between Kondo screening, and AF or modulated SL ordering. Our systematic approach starts from an arbitrary fixed value of electronic filling $n_{c}$. The pure Kondo lattice part of the Hamiltonian (1) $H_{\mathrm{KL}}$ is thus roughly characterized by one energy scale [63]: the noninteracting Kondo temperature $T_{K 0}$. Doniach's argument [8] can be reproduced phenomenologically within a 


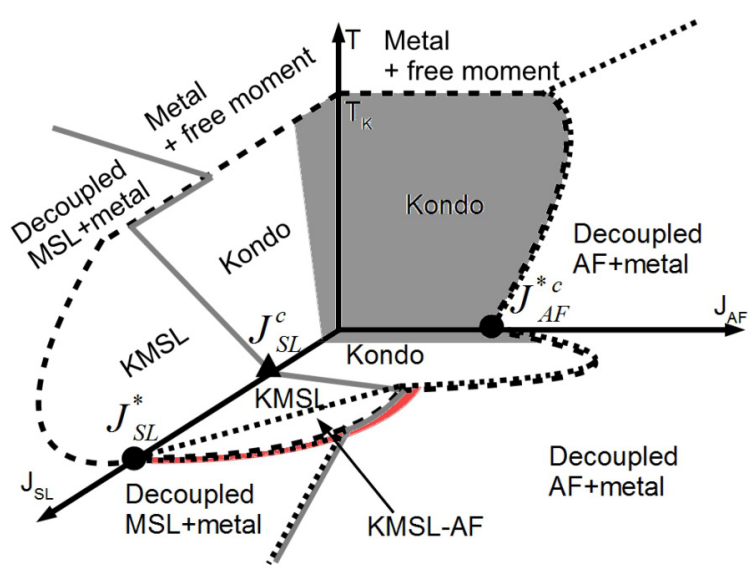

FIG. 1. (Color online) Schematic phase diagram of the KondoHeisenberg model in parameter space $\left(T, J_{\mathrm{AF}}, J_{\mathrm{SL}}\right)$, showing the MSL phase transition (solid line), the AF phase transition (dotted line), and the Kondo crossover (dashed line). Three specific QCPs are specifically marked: two of them indicating a breakdown of Kondo effect (circle) and the third one indicating the onset of the MSL (triangle) in a Kondo regime. The gray area defines the Kondo phase with $\phi_{0}=0$. The mean-field transition separating the Kondo phase from the light metal is expected to become a crossover beyond the mean-field. The Kondo to KMSL phase, the KMSL to MSL, the KMSL-AF to MSL, metal to MSL, metal to AF, and the the KMSL-AF to KMSL are second-order phase transitions. The Kondo to AF phase and the KMSL-AF to AF are first-order phase transitions. The red area defines the MSL-AF coexisting phase. The various phases and their related order parameters are defined in Sec. III A.

Kondo-Heisenberg model by comparing $T_{K 0}$ with the energy scale that characterizes the Heisenberg part $H_{\mathrm{RKKY}}$ of the Hamiltonian. Therefore, in this section we fix the $T_{K 0}$ that provides the energy unit scale of the problem, and we analyze the phase diagram as a function of the two RKKY phenomenological energies $J_{\mathrm{AF}}$ and $J_{\mathrm{SL}}$, and the temperature $T$. The resulting phase diagram is depicted schematically in Fig. 1, on the basis of the numerical solution of the self-consistent mean-field relations shown in Fig. 2.

\section{A. Description of phases and order parameters}

The schematic $\left(T, J_{\mathrm{AF}}, J_{\mathrm{SL}}\right)$ phase diagram of the model is depicted on Fig. 1. As expected, the Kondo regime is obtained at low temperature for sufficiently small RKKY energies. In contrast, pure RKKY-dominated phases appear when $J_{\mathrm{AF}}$ or $J_{\mathrm{SL}}$ are large enough. At intermediary RKKY coupling regime, the general phase diagram (see Fig. 1) also reveals coexisting phases between the Kondo and MSL orders (KMSL) and the Kondo phase, a MSL order and an AF order (KMSL-AF). Note that the AF order can coexist with the Kondo effect only in the presence of the MSL order.

Leaving aside the issue of superconductivity, it thus appears in accordance with Doniach's view [8] there are two different kinds of QCPs. In one of them, the order parameter characterizing the traditional QCP (denoted by $\mathrm{QCP}^{c}$ ) is the local magnetization, which can be easily measured experimentally. The second kind of QCP (denoted by $\mathrm{QCP}^{\star}$ ) marks the breakdown of the Kondo effect, which is also called fractionalization, and
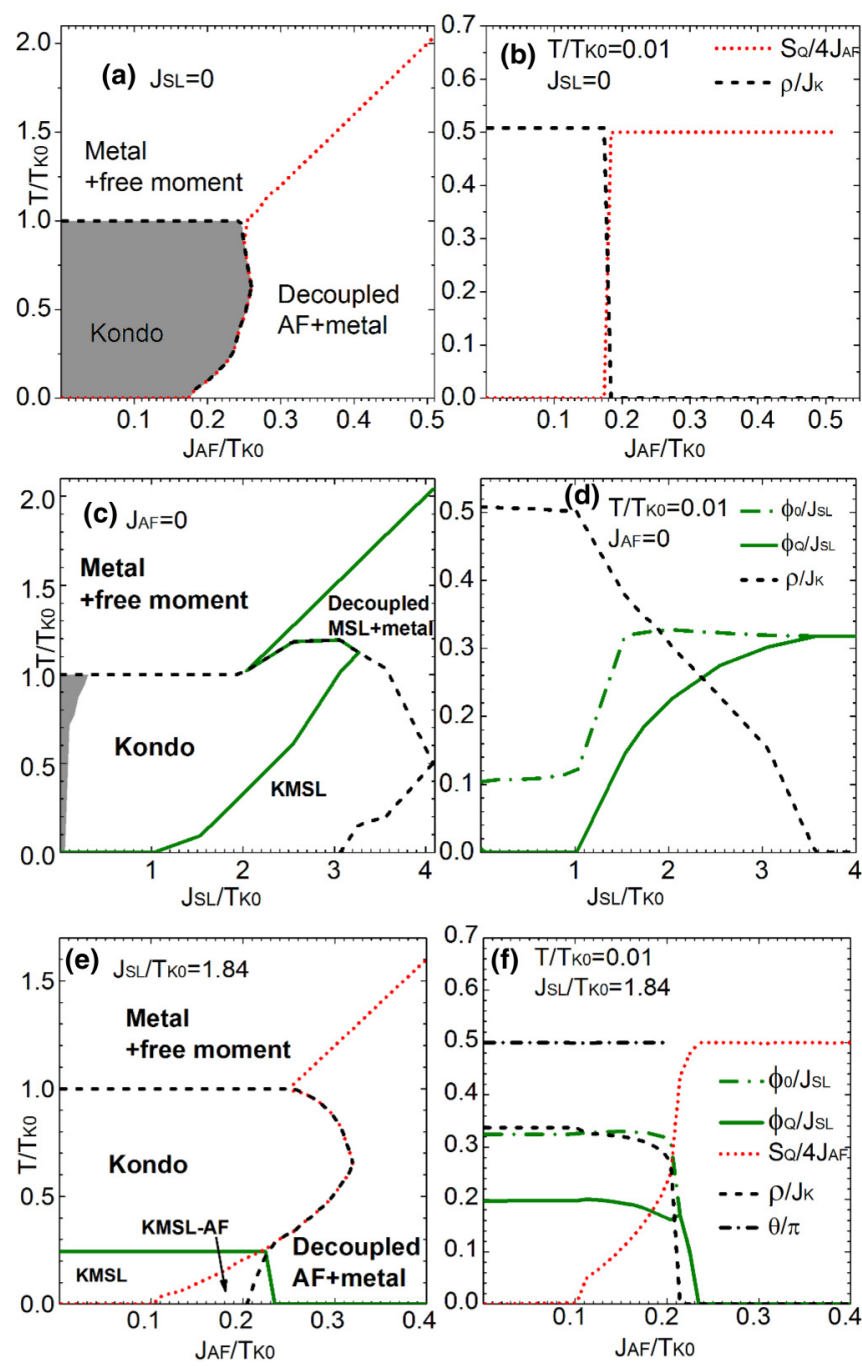

FIG. 2. (Color online) Phase diagrams [(a), (c), (e)] and the variations of the mean-field parameters at $T / T_{K 0}=0.01[(\mathrm{~b}),(\mathrm{d})$, (f)] for $J_{K} / t_{c}=0.8686$, the conducting electron density $n_{c}=0.7$, and $T_{K 0} / t_{c}=0.098$ where the MSL phase transition is represented by the solid line, the AF phase transition is indicated by the dotted line, and the Kondo crossover is depicted in the dashed line. We choose the regime $J_{\mathrm{SL}}=0$ as a function of $J_{\mathrm{AF}}$ in (a) and (b). We show the regime $J_{\mathrm{AF}}=0$ as a function of $J_{\mathrm{SL}}$ in (c) and (d) and, finally, we consider the intermediary case $J_{\mathrm{SL}}^{c}<J_{\mathrm{SL}}<J_{\mathrm{SL}}^{\star}$ as a function of $J_{\mathrm{AF}}$ in (e) and (f). The gray area delimits the Kondo phase with $\phi_{0}=0$.

has been first discussed from a theoretical point of view by several authors [7,64-71]. Testing the vanishing of the Kondo effective coupling and considering the RKKY-dominated order parameters, the phase diagram also reveals a $\mathrm{QCP}^{\star}$ and two $\mathrm{QCPs}^{c}$ at $J_{\mathrm{SL}}^{\star}, J_{\mathrm{SL}}^{c}$, and at $J_{\mathrm{AF}}^{\star c}$, respectively, which will be discussed further.

Among the five mean-field parameters that have been introduced, $\phi_{Q}, S_{Q}$, and $\theta$ correspond to true order parameters characterizing phase transitions, while $\phi_{0}$ and $\rho$ rather characterize crossovers to correlated regimes, which are associated, respectively, with the spin-liquid and the Kondo heavy-fermion phases. Hereafter, we classify these phases in four sets: the disordered phase, the pure AF, the pure MSL, and the mixed $\mathrm{AF}+\mathrm{MSL}$ ordered phases. 


\section{Disordered phase}

The disordered phase covers the paramagnetic and metallic at high-temperature phase and the Kondo correlated heavyfermion phases. Within our mean-field approach, the crossover Kondo temperature is signaled by a transition at $T_{K}$ which is characterized by the continuous vanishing of the Kondo parameter the Kondo regime being realized for $\rho \neq 0$ for $T<T_{K}$. Below $T_{K}$, the mean-field approach may distinguish two kinds of Kondo regimes, depending on the value of the homogeneous SL parameter $\phi_{0}$. The Kondo regime with a finite $\phi_{0}$ does not break any symmetry.

a. The decoupled regime. This regime is established for a temperature higher than all the interaction energy scales, i.e., when $T>T_{K 0}, J_{\mathrm{SL}}, J_{\mathrm{AF}}$. At the mean-field level, this corresponds to a complete decoupling between the Kondo spins and the conduction electrons, with $\phi_{0}=\phi_{Q}=S_{Q}=\rho=0$. The transport and thermodynamic properties correspond to that of a light metal, and the resulting magnetic susceptibility can be directly associated with free moments.

$b$. The Kondo regime with $\phi_{0}=0$. This usual Kondo regime is defined by the condition $\rho \neq 0$, with all other mean-field parameters being nullified. It is obtained for $T<T_{K}$ when $J_{\mathrm{SL}} \approx 0$, and it extends to $J_{\mathrm{AF}}<J_{\mathrm{AF}}^{\star c}$ as shown in Fig. 2 .

c. The Kondo regime with $\phi_{0} \neq 0$. Finite values of both $\phi_{0}$ and $\rho$ characterize this correlated heavy-fermion regime which does not break any symmetry $\left(\phi_{Q}=S_{Q}=0\right)$. Note that the solutions $\theta=-\pi / 2$ with $\phi_{0}>0$ and $\theta=0$ with $\phi_{0}<0$ are equivalent to each other. The apparent symmetry breaking provided by $\theta=-\pi / 2$ can be recovered by the local gauge transformation $\left(\phi_{0}>0, \theta=-\pi / 2\right) \rightarrow\left(\phi_{0}<\right.$ $0, \theta=0)$. Physically, this corresponds to a Kondo coupling between conduction electrons and local moments dispersion of opposite sign.

\section{Pure AF phase}

The AF phase is obtained at low temperature for $J_{\mathrm{AF}}>J_{\mathrm{AF}}^{\star c}$. Here, the occurrence of long-range magnetic AF ordering coincides with the suppression of the Kondo effect. This decoupled $\mathrm{AF}+$ metal phase is characterized within our meanfield approach, by a finite staggered magnetization $S_{Q}$ together with $\phi_{0}=\phi_{Q}=\rho=0$. We find no coexistence between the Kondo effect and the AF order, but such coexisting phases might appear in different lattice structures. The pure AF phase breaks lattice and time-reversal symmetries.

\section{MSL phases}

The MSL order parameter merges with the mean-field $\phi_{Q}$ which was introduced in $[49,50]$ within the purely Heisenberg models. Here, the presence of conduction electrons and their Kondo coupling to the local moments lead to two MSL regimes:

a. The pure, decoupled, MSL phase. This phase is a purely RKKY-dominated phase characterized by a finite MSL order parameter $\phi_{Q}$, with no Kondo effect $(\rho=0)$, and without an $\mathrm{AF}$ order $\left(S_{Q}=0\right)$. Here, the conduction electrons are effectively decoupled from the Kondo spins forming the MSL order. The lattice translation symmetry is broken but the local magnetization remains equal to zero.
The $Z 4$ lattice symmetry observed in the $\mathrm{URu}_{2} \mathrm{Si}_{2}$ compound is also broken as discussed in Ref. [49]. Note that the $Z 4$ symmetry breaking has been observed in magnetic susceptibility [72] and cyclotron resonance [73] experiments. In view of that, the authors indicate the existence of a nematic order in the [110] direction. Here, the symmetry group of the square lattice is very different from the real case appropriate for this compound. Therefore, in this case, the $Z 4$ symmetry breaking cannot be the same as in the realistic 3D lattice. However, this symmetry breaking is a particular property of the $\mathrm{HO}$ and the MSL phases in the spirit of what was developed earlier in Ref. [48].

b. The Kondo-MSL phase. The Kondo and the MSL phases merge together at low temperature for a broad range of intermediate couplings $J_{\mathrm{SL}}^{c}<J_{\mathrm{SL}}<J_{\mathrm{SL}}^{\star}$. Inside this phase, conduction electrons are strongly correlated to the MSL ordering formed by the local moments. Within our mean-field approach, this phase is defined by a finite Kondo parameter $\rho$ coexisting with a finite MSL order parameter $\phi_{Q}$. Here, the order is purely MSL, which means that the system undergoes no staggered magnetization, i.e., $S_{Q}=0$. We find that the Kondo effective hybridization is inhomogeneous, with $\theta=-\pi / 2$.

Such a phase modulation in the Kondo parameter results from the lattice symmetry breaking of the MSL. It is still compatible with the local U(1) gauge invariance mentioned in our mean-field approach. Indeed, without the MSL order, any local phase variation of the Kondo hybridization could be gauged out invoking a local U(1) transformation. However, in the presence of an MSL order, such a local gauge transformation for the Kondo term would also have some effect on the intersite spin-liquid term. Using appropriate local gauge transformations, one might thus be able to map a set of mean-field solutions $\theta=-\pi / 2$ to $\theta=0$ but at the same time $\phi_{0}$ and $\phi_{Q}$ must be mapped to $-\phi_{0}$ and $-\phi_{Q}$, respectively. This strongly correlated phase breaks the square lattice translation and the $Z 4$ symmetries.

\section{Mixed AF + MSL phase}

a. The decoupled $A F+M S L$ phase. When $J_{\mathrm{AF}}$ and $J_{\mathrm{SL}}$ are of similar magnitude and are both much larger than $T_{K 0}$, the system forms an RKKY-dominated phase with the conduction electrons effectively decoupled from the local moments. This AF + MSL decoupled phase is defined by finite $S_{Q}$ and $\phi_{Q}$, together with $\rho=0$. This manifests itself in a very narrow part of the phase diagram. Such a phase breaks time reversal symmetry as well as the lattice translation and the $Z 4$ symmetries.

b. The Kondo coupled AF+MSL phase. This Kondo coupled AF and MSL phase (KMSL-AF) is characterized by nonzero values of all the considered mean-field parameters $\phi_{Q}, \phi_{0}, S_{Q}$, and $\rho$. It is realized when the three relevant energy scales $T_{K 0}, J_{\mathrm{AF}}$, and $J_{\mathrm{SL}}$ are of the same order of magnitude. This phase breaks the same symmetries as the decoupled AF + MSL phase.

\section{B. Numerical results}

a. The Kondo-AF order competition. For $J_{\mathrm{SL}}=0$, the Kondo and AF order competition exhibits a QCP at $J_{\mathrm{AF}}^{\star c}=$ $0.2 T_{K 0}$ [see Fig. 2(a)] which is both a $\mathrm{QCP}^{\star}$ (breaking the 
Kondo effect) and a $\mathrm{QCP}^{c}$ (onset of long-range magnetic order). The magnitude of $J_{\mathrm{AF}}^{\star c}$ is of the same order as $T_{K 0}$ and this is consistent with Doniach's arguments. The Kondo to AF transition is of first-order nature as emphasized by the discontinuities of the effective Kondo coupling $\rho$ and the staggered magnetization field $S_{Q}$ at the transition [see Fig. 2(b)]. The Kondo to normal metal phase transition manifests itself as a second-order phase transition because of the mean-field approximation but it might indeed be a crossover instead. The AF to metal phase transition is second order in our case. Note that the mean-field approximation does not always describe the right order of the phase transitions. The investigation of these features beyond mean field can be done by standard calculations of the fluctuations on the Weiss, Kondo [57], and spin-liquid mean fields. The consideration of those fluctuations goes much beyond the goal of this paper and is left aside for later work.

b. The Kondo-MSL order competition. For $J_{\mathrm{AF}}=0$, both the Kondo local and spin-liquid intersite screening reveal two QCPs [see Fig. 2(c)]: a $\mathrm{QCP}^{c}$ at $J_{\mathrm{SL}}=T_{K 0}$ and a $\mathrm{QCP}^{\star}$ at $J_{\mathrm{SL}}^{\star}=3 T_{K 0}$. The $\mathrm{QCP}^{c}$ at $J_{\mathrm{SL}}=T_{K 0}$ characterizes the secondorder phase transition from the Kondo phase with $\phi_{0} \neq 0$ to the KMSL phase hybrid phase as shown by the continuity of the modulated spin-liquid field $\phi_{Q}$ at the transition [see Fig. 2(d)]. The $\mathrm{QCP}^{\star}$ at $J_{\mathrm{SL}}=3 T_{K 0}$ appears at the KMSL to MSL second-order phase transition as emphasized by the continuous vanishing of the effective Kondo field $\rho$ at the transition. In the MSL phase, the homogeneous and modulated spin-liquid mean-field order parameters $\phi_{0}$ and $\phi_{Q}$ are of the same magnitude, i.e., $\phi_{0}=\phi_{Q}$. This is related to the absence of the second-nearest-neighbor coupling and it was first noted in our earlier work [49]. The metal to Kondo and the Kondo with $\phi_{0}=0$ to Kondo with $\phi_{0} \neq 0$ changes of state appear again as an artifact of our mean-field treatment but they are both, in fact, crossovers. The metal to the MSL phase transition is second order. In contrast, the change of state from the Kondo with $\phi \neq 0$ state to the MSL phase is a first-order phase transition.

c. The Kondo-MS-AFL order competition. The KMSL-AF phase appears at an intermediate SL coupling $J_{\mathrm{SL}}^{c}<J_{\mathrm{SL}}<J_{\mathrm{SL}}^{c}$ as shown in Figs. 1 and 2(e). In the KMSL-AF phase, the staggered magnetization $S_{Q}$ increases with the AF coupling as shown in Fig. 2(f). The phase transition between the KMSL and the KMSL-AF phases is second order and this is signaled by the continuous change in the magnitude of $S_{Q}$ as shown in Fig. 2(f). However, the transition from the KMSL-AF to the MSL + AF phases and from the MSL + AF to AF phases are both first order as attested by the discontinuities of the mean-field order parameters $\phi_{0}, \phi_{Q}$, and $S_{Q}$ at the transitions [see Fig. 2(f)].

We note the existence of the MSL + AF coexisting phase in a very narrow part of the phase diagram (between the KMSL$\mathrm{AF}$ and AF phases). This coexisting phase disappears for larger values of $J_{\mathrm{SL}}$ and temperature.

The $\theta$ phase is equal to $-\pi / 2$ in the KMSL and KMSL-AF phases and vanishes with the effective Kondo mean field $\rho$. The metal to Kondo phase change is a crossover while the Kondo to KMSL and the metal to AF changes of state are real second-order phase transitions. Finally, the Kondo with $\phi_{0} \neq 0$ to AF order is a first-order phase transition.

\section{Discussion}

Among the well-accepted scenarios explaining pressuredriven QCPs in heavy fermions, the general Doniach's argument [8] relies on the competition between local Kondo screening and intermoment Ruderman-Kittel-Kasuya-Yosida (RKKY) magnetic interaction [74-76]. When the Kondo coupling dominates the RKKY mechanism, the thermodynamic, transport, and magnetic properties are characterized by a Fermi liquid behavior with a large effective mass [77-79]. The opposite regime leads generally to a magnetically ordered ground state or, alternatively, to a spin-liquid (SL) phase [60] in a frustrated system $[64,65]$.

In our model, two quantum critical lines are seen in the (JAF, JSL) phase diagram, ending on three QCPs when either JAF or JSL reaches zero. One line, denoted by a $\star$, marks the Kondo breakdown. The other critical line, with a $c$ index, characterizes the breaking of the lattice translation symmetry. A $\mathrm{QCP}^{\star c}$ appears at the Kondo-AF phase transition $\left(J_{\mathrm{SL}}=\right.$ $\left.0, J_{\mathrm{AF}}=0.2 T_{K 0}\right)$ and a $\mathrm{QCP}^{\star}$ occurs at the KMSL to MSL phase transition for $\left(J_{\mathrm{SL}}=3 T_{K 0}, J_{\mathrm{AF}}=0\right)$. Moreover, a QCP separates the Kondo and KMSL phases $\left(J_{\mathrm{SL}}=T_{K 0}, J_{\mathrm{AF}}=0\right)$.

A QCP $^{\star}$ separates a heavy Fermi liquid phase where the conventional Luttinger theorem [80-82] holds, and a fractionalized phase in which such a theorem does not apply any longer. In other words, the number of quasiparticles in the heavy Fermi liquid phase is given by the sum of the conduction electrons and the localized electrons. On the contrary, light conduction electrons only provide the metallic properties of the fractionalized phase. From this theoretical definition emerged the following experimental signature of a Kondo breakdown $\mathrm{QCP}^{\star}$ in a periodic system: the Fermi surface is expected to vary from a large to a small volume as the $\mathrm{QCP}^{\star}$ is crossed from the Kondo to the RKKY phase. Other signatures of a $\mathrm{QCP}^{\star}$ are also expected for crystals as well as for nonperiodic systems, including, for example, the change of sign in the Hall constant (see, for example, [83]). This mechanism drives the physics in the vicinities of both the $\mathrm{QCP}^{\star}\left(J_{\mathrm{SL}}=3 T_{K 0}\right)$ and the $\mathrm{QCP}^{\star c}\left(J_{\mathrm{AF}}=0.2 T_{K 0}\right)$.

Thereby the volume of the Fermi surface is expected to have a significant discontinuity when crossing a $\mathrm{QCP}^{\star}$. One may naively think that this single feature would be enough to provide a clear experimental signature of the Kondo breakdown transition. However, experimental reality is more complex than that since most of the (magnetic) QCPs ${ }^{c}$ also break lattice translation symmetry. This also leads to another significant variation of the Fermi surface due to the folding of the first Brillouin zone. Signatures of these quantum phase transitions can still be obtained from a Fermi surface analysis, but this requires a careful investigation which takes into consideration the volume as well as nesting and symmetry properties.

Moreover, we have now two situations where the lattice symmetry breaking $\left(\mathrm{QCP}^{c}\right)$ might coincide or not with the Kondo breakdown $\left(\mathrm{QCP}^{\star}\right)$. This issue extends to the MSL order the general question as to whether the Kondo effect may coexist or not with magnetic ordering. In particular, here we find that for the AF transition the Kondo breakdown coincides with lattice symmetry breaking $\left(\mathrm{QCP}^{c}=\mathrm{QCP}^{\star}\right)$. This means that the local Kondo screening disappears precisely when the 
magnetic moments order sets in. For the MSL transition, we find a Kondo breakdown inside the MSL phase.

Possible coexistence of Kondo effect and magnetic order has been analyzed as a function of several model parameters including the nature of magnetic ordering, and on the dimensionality of the physical system [84].

From these previous analyses we may deduce that the phase diagram obtained from a 3D model would still present a Kondo-MSL phase but it would probably be extended by a supplementary Kondo-AF phase. It would be interesting to test this issue by doing, for instance, optical conductivity measurements under pressure. The Kondo hybridization gap is expected to be maintained at a transition which preserves Kondo effect, as observed for the HO transition. Knowing whether it is also preserved at the AF transition would provide a clear signature of the possible Kondo-AF coexistence.

\section{CASE OF URu $\mathrm{Si}_{2}$}

In this section, we use the formalism developed in Secs. II and III to analyze the particular case of $\mathrm{URu}_{2} \mathrm{Si}_{2}$. The MSL model assumes that the $\mathrm{HO}$ order parameter has both local and itinerant characteristics $[49,50]$. Applying the MSL model to the $\mathrm{HO}$ phase of $\mathrm{URu}_{2} \mathrm{Si}_{2}$, we reproduce qualitatively different experimental observations such as the entropy quenching, the $(\mathrm{T}, \mathrm{P})$ phase diagram, the MSL gap evolution, and the density of state.

In the following, we set the Kondo coupling $J_{K}=54.37$ $\mathrm{meV}$ and the conduction electron hopping $t_{c}=63.31 \mathrm{meV}$ to establish a Kondo temperature around $T_{K}=70 \mathrm{~K}$. This value of the Kondo temperature $T_{K}$ is suggested by resistivity measurements [14,23] and by optical conductivity $[29,85]$ experiments. Hall experiments $[86,87]$ and ARPES $[27,28,88-$ 90] measurements also confirm the existence of hybridized bands.

We adjust the spin-liquid coupling at $J_{\mathrm{SL}}=11.37 \mathrm{meV}$ to settle the $\mathrm{HO}$ phase transition temperature $T_{0}=17.5 \mathrm{~K}$ as indicated in the specific-heat data [12]. Note that the tuning of $J_{\mathrm{AF}}$ reproduces the pressure tuning given by the experiments [50].

\section{A. Variation of entropy and specific heat}

\section{Main experimental results}

The hidden order phase is signaled by a large jump in the specific heat at $17.5 \mathrm{~K}[12,18]$ characterizing a second-order phase transition. The total entropy quenched in this phase is around $0.3 k_{B} \ln (2)$ per $\mathrm{U}$ atoms [12,14]. In spite of this entropy quenching,the magnetic moment initially measured in Ref. [15] remains very small. The magnetic moment per $\mathrm{U}$ atoms is no longer intrinsic of $\mathrm{HO}$ phase since it is inhomogeneous [91,92] and it strongly depends on the sample's purity and stress distribution (see Sec. III, p. 1306 of Ref. [18]). Moreover, the value of the magnetic moment vanishes in a high-purity compound $[93,94]$. Consequently, the HO phase cannot be explained by a small magnetization antiferromagnetic phase (see discussion in Sec. III, p. 1306 of Ref. [18]).
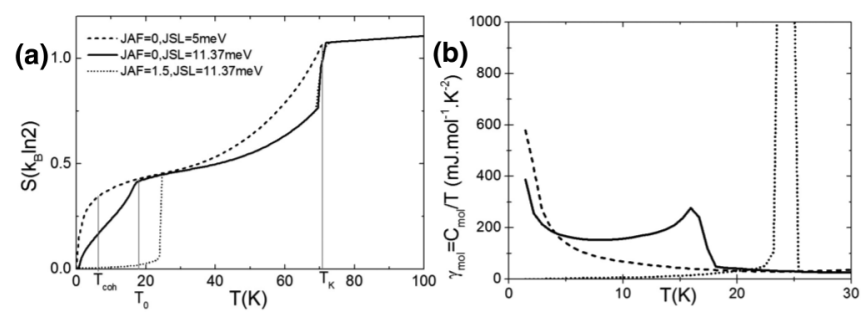

FIG. 3. Temperature dependence of (a) the entropy, (b) the Sommerfeld coefficient per mole $\gamma_{\mathrm{mol}}=C_{\mathrm{mol}} / T$, for a pure Kondo ground state (dashed line, $J_{\mathrm{SL}}=5 \mathrm{meV}$ and $J_{\mathrm{AF}}=0$ ), a Kondo-MSL ground state (solid line, $J_{\mathrm{SL}}=11.37 \mathrm{meV}$ and $J_{\mathrm{AF}}=0$ ), and an AF ground state (dotted line, $J_{\mathrm{SL}}=11.37 \mathrm{meV}$ and $J_{\mathrm{AF}}=1.5 \mathrm{meV}$ ). Note that the entropy evolution around $T_{K}$ is abrupt because of the mean-field approximation for the Kondo interaction. A crossover is expected around $T_{K}$ beyond the mean field. We clearly see that the Kondo-MSL scenario, unlike the pure Kondo one, is characterized by a peak in $C / T$ around $T=17 \mathrm{~K}$. Note that the extremely sharp peak obtained at the Néel temperature within the AF scenario (dotted line) should be broadened within a more realistic three-dimensional lattice (see the text). Notice that at very low temperature, the Sommerfeld coefficient is expected to saturate and reach its zero-temperature limit $1 / T_{\text {coh }}$ (not presented here).

\section{Numerical results}

The entropy $S$ per site, with $S=-\partial \mathcal{F}_{\mathrm{MF}} / \partial T$, and the Sommerfeld coefficient per U atoms $\gamma=C / T=\partial S / \partial T$ are presented in Figs. 3(a) and 3(b) for the three following cases: a pure Kondo phase with $T_{K}=70 \mathrm{~K}$ (dashed line), a Kondo + $\phi_{0}$ phase with $T_{K}=70 \mathrm{~K}$, and MSL order with $T_{0}=17,5 \mathrm{~K}$ (solid line) and a Kondo $+\phi_{0}$ phase with $T_{K}=70 \mathrm{~K}$ and $\mathrm{AF}$ order with $T_{N}=24 \mathrm{~K}$ (dotted line). These three cases allow us to compare the effect of each order parameter on the thermodynamic properties. The Sommerfeld coefficient per mole is $\gamma_{\mathrm{mol}}=C_{\mathrm{mol}} / T$, where $C_{\mathrm{mol}}$ is the specific heat per mole. The specific heat per mole $C_{\mathrm{mol}}$ is connected to the entropy per $\mathrm{U}$ atoms $S$ by the relation $C_{\mathrm{mol}}=T N_{A} \partial S / \partial T$ with $N_{A}$ being the Avogadro number.

In the three cases, the entropy is quenched around the Kondo temperature $T_{K} \approx 70 \mathrm{~K}$ giving $S=k_{B} \ln 2$. The small difference in entropy with $k_{B} \ln 2$ at $T_{K}$ in Fig. 3 originates the entropy of the conduction electrons since not all of them are frozen at $T_{K}$. Note that the abrupt decrease of entropy around $T=T_{K}$ is an artifact of the mean-field approximation describing the Kondo crossover as a phase transition.

In the case of a pure Kondo phase [dashed line on Fig. 3(a)], the entropy below $T_{K}$ presents two different regimes of decreasing. The first regime occurs around $T_{K}$ and is related to the local Kondo screening. The second regime, occurring for $T<T_{\text {coh }}$, is related to the coherence of the associated Fermi liquid state.

As defined in Refs. [63,95], $T_{\text {coh }}$ is related to the formation of a coherent Fermi liquid state with coherent quasiparticles and a strong enlargement of Fermi surface including both the local moments and the conduction electrons. The coherence temperature is defined as $T_{\text {coh }} \equiv \frac{1}{\gamma(T=0)}$. The appearance of the coherent Fermi liquid manifests itself with a smooth crossover at $T_{\text {coh. }}$. Below $T_{\text {coh }}$, the entropy decreases linearly to zero. 
The different variation of the entropy between the pure Kondo (dashed line) and Kondo with $\phi_{0}$ phases [solid and dotted lines on Fig. 3(a)] can be explained by the spin-liquid correlations appearing with the Kondo screening at $T_{K}$ which contributes to freeze a part of that entropy. Note that in the model presented here, we do not take into account the orbital symmetry of the uranium atoms and the crystal field splitting that also contributes to the determination of $T_{K}$.

As for the Kondo to KMSL phase transition, the variation of entropy takes place at $T_{0}$ [see solid line in Fig. 3(a)]. This change can be related to a jump in specific heat as observed in real compound [12,23]. Moreover, the variation of entropy between $T_{0}$ and $T=0 \mathrm{~K}$ is around $0.3 k_{B} \ln 2$ which is close to the value measured in the bulk compound $[12,14]$. This variation is also seen at the Kondo-AF transition [dotted line in the Fig. 3(a)] and this suggests a similar mechanism for this entropy quench between the KMSL and AF phases.

The coherence temperature of the Kondo lattice $T_{\text {coh }}$ is smaller than the KMSL phase transition temperature $T_{0}$. This implies that the Kondo screening is not yet complete at $T_{0}$. We argue that the partial Kondo coupling existing above $T_{0}$ might explain the band-structure reconfiguration observed around $30 \mathrm{~K}$ in optical conductivity $[30,31]$ and ARPES experiments [88]. The description of the Kondo screening within a simple coupling to an itinerant band is a strong feature of our model.

The Sommerfeld coefficient $\gamma_{\mathrm{mol}}=C_{\mathrm{mol}} / T=\partial S / \partial T$ is presented in Fig. 3(b). We see a jump in the specific heat at the Kondo to KMSL transition (solid line) and at the Kondo to AF transition (dotted line) corresponding to a second-order phase transition. The jump in $\gamma_{\mathrm{mol}}$ appears around $200 \mathrm{~mJ} \mathrm{~mol}^{-1} \mathrm{~K}^{-2}$ and this is comparable with the experimental value [12].

From the three curves of the Fig. 3(b), we show that Kondo lattice Fermi liquid coherence by itself cannot reproduce a peak in $C / T$ around $17 \mathrm{~K}$. In spite of the existing Kondo regime, it is necessary to add a RKKY-type mechanism, associated with the AF or the MSL order, to reproduce this peak.

The Sommerfeld coefficient peak value at the AF phase transition is higher than in the real compound. This results from the square lattice approximation in which the Kondo breakdown coincides with AF order. We are aware that a three-dimensional model might lead to a coexistence between Kondo and AF; in that case, we could expect the peak in $C / T$ to be less sharp at the AF transition. Note that the peak in the Sommerfeld coefficient appearing below $T_{0}$, around $T \approx T_{\text {coh }}$, characterizes the formation of the coherent state constituting the Fermi liquid. This peak does not appear in the real compound where a signature of a superconducting state is observed.

\section{B. The (T,P) phase diagram and the evolution of the Fermi surface \\ 1. Main experimental results}

The temperature-pressure phase diagram was extracted from neutron scattering data $[19,20,94]$ and from resistivity measurements [23]. It shows a second-order HO phase transition at $T=17,5 \mathrm{~K}$ at ambient pressure. However, it has been shown that the HO phase suffers a first-order phase transition to the AF phase with magnetic moment $m=$

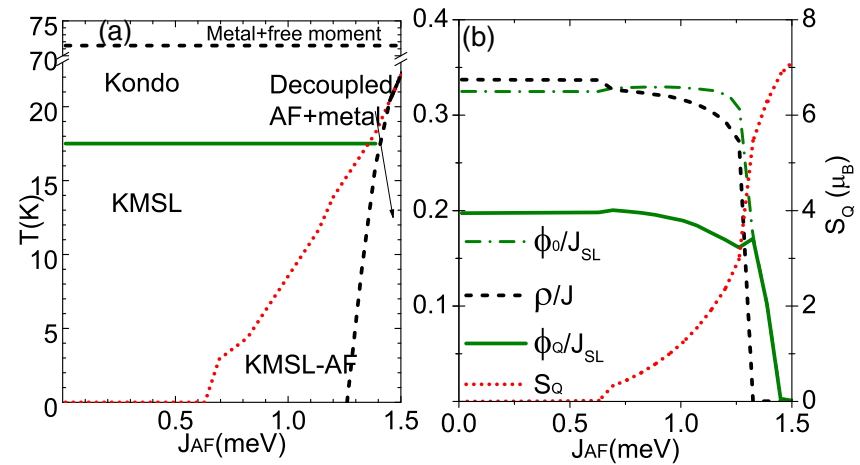

FIG. 4. (Color online) (a) $\left(T, J_{\mathrm{AF}}\right)$ phase diagram in $\mathrm{URu}_{2} \mathrm{Si}_{2}$ with $J_{\mathrm{SL}}=11,37 \mathrm{meV}, J_{K}=54,37 \mathrm{meV}$, and $t_{c}=63,31 \mathrm{meV}$. Here, $J_{\mathrm{AF}}$ represents phenomenologically the effect of pressure. (b) Evolution of the mean-field parameter and the staggered magnetization $S_{Q}$ for the ground state. In both figures, $J_{\mathrm{SL}}=11,37 \mathrm{meV}$, $J_{K}=54,37 \mathrm{meV}$, and $t_{c}=63,31 \mathrm{meV}$.

$\mu_{B}$ at $P=0.5 \mathrm{GPa}[19,20,23,94]$. Conductivity measurements [14,96], ARPES [28], infrared spectroscopy [30,31], Hall effect [86,87], and STM [25] show a gap opening at the Fermi surface inducing a Fermi surface reconstruction through the HO phase transition. Moreover, the Fermi surface and transport properties exhibit strong similarities in both the $\mathrm{HO}$ and the AF phases [97].

\section{Numerical results}

The $\left(T, J_{\mathrm{AF}}\right)$ phase diagram presented in Fig. 4 shows a second-order phase transition between the Kondo and the KMSL phases and a first-order transition between the KMSL$\mathrm{AF}$ and AF phases associated to the Z4 symmetry breaking.

This corroborates with the INS experiments $[19,20,94]$. The KMSL phase breaks the $Z 4$ and lattice symmetries while the AF phase breaks the time-reversal and the lattice symmetries. The transition from the KMSL to KMSL-AF is associated with the time-reversal and $Z 4$ symmetry breakings. Note that the KMSL phase shows no sign of existence of a magnetic moment as expected in the realistic $\mathrm{HO}$ phase (see discussion in Sec. IV A 1).

Contrary to what is observed experimentally, the phase diagram presented in Fig. 4 exhibits an extra KMSL-AF coexisting phase. This KMSL-AF phase discussed in Sec. III A 4 b breaks the time-reversal symmetry and restores progressively the $Z 4$ symmetry. This KMSL-AF phase appears in a region of the phase diagram where all the couplings have similar values and this might imply the presence of strong fluctuations. A more detailed treatment of the frustration and fluctuations on the realistic bct lattice will be necessary to conclude on the stability on this coexisting phase. This study is initiated in Ref. [51].

We observe a strong Fermi surface reconstruction with folding at the Kondo-KMSL phase transition (at $T_{0}=17.5 \mathrm{~K}$ ) [see Figs. 5(e) and 5(c)]. Note that in our model, this reconstruction is associated with the $Z 4$ and lattice symmetry breaking appearing simultaneously with the MSL order parameter.

As displayed by quantum oscillation experiments [97], the $\mathrm{HO}$ and AF phases exhibit similar Fermi surfaces. This similarity results from a folding of the initial Fermi surface 

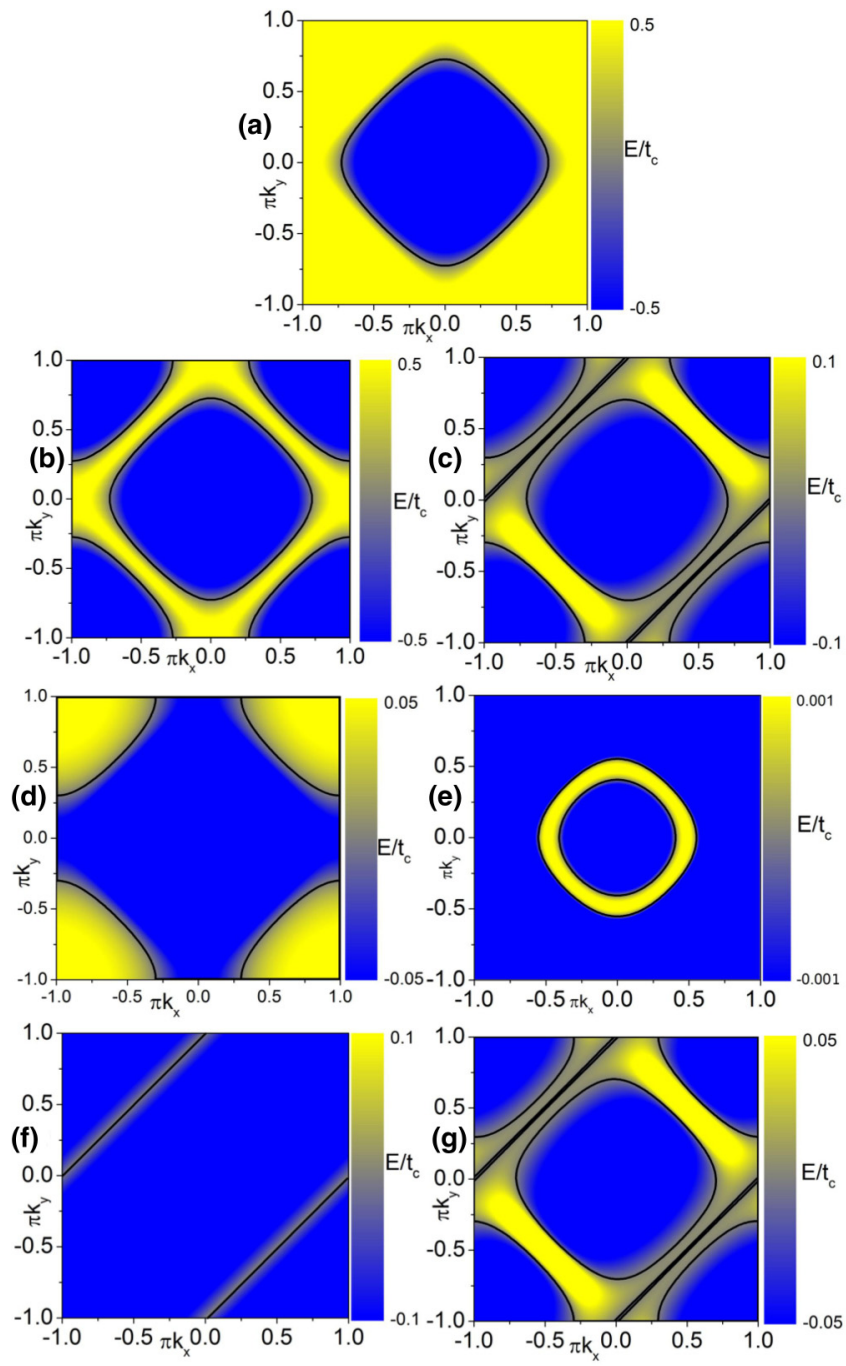

FIG. 5. (Color online) Dispersions of (a) the paramagnetic metal phase, (b) the AF phase, (c) the KMSL phase, (d) the Kondo phase with $\phi=0$ and with (e) $\phi \neq 0$, (f) the spinons of the MSL phase, and (g) the KMSL-AF phase in the first Brillouin zone. The solid line represents the Fermi surface at the Fermi level $(E=0)$. In (e), the hole states are confined between the two solid lines. The similarity between the AF (b) and MSL (c) folded Fermi surfaces is consistent with quantum oscillation experiments realized under pressure [97].

[see Figs. 5(a), 5(b), and 5(c)]. Moreover, in our model, this similarity is emphasized by the progressive appearance of an AF order in the KMSL phase that steadily destroys the Z4 symmetry breaking [see Figs. 5(b), 5(c), and 5(g)]. The realistic Fermi surface of $\mathrm{URu}_{2} \mathrm{Si}_{2}$ produces four small pockets in the [100] and [010] directions. In our model, the MSL order produces a small FS along the [100] to [010] directions. Considering a second-next-nearest neighbor term in the electronic spectrum, it might be possible to produce some extra hole and electron pockets in the FS along these directions. However, this feature has to be confirmed considering a more realistic band dispersion in the 3D model. A detailed analysis of the Fermi surfaces presented in Fig. 5 is given in Appendix B.
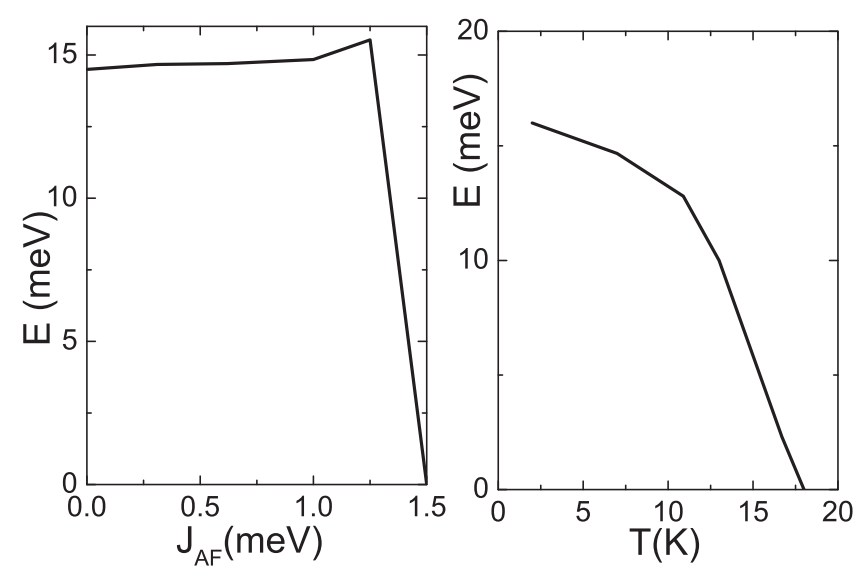

FIG. 6. (a) Direct gap at the $X_{1}$ point as a function of $J_{\mathrm{AF}}$ for the ground state. (b) Direct gap at the $X_{1}$ point as a function of the temperature in the KMSL state $\left(J_{\mathrm{AF}}=0.31 \mathrm{meV}\right)$.

\section{Evolution of the MSL gap \\ 1. Main experimental results}

Inelastic neutron scattering experiments exhibit a resonance at the commensurate wave vector $Q_{0}=\frac{2 \pi}{a}(1,0,0)$ which transforms itself into a strong elastic $\mathrm{AF}$ signal for a pressure $P>5$ kbar [21,22]. Another inelastic resonance occurs in the $\mathrm{AF}$ and $\mathrm{HO}$ phases at the incommensurate wave vector $Q^{*}=\frac{2 \pi}{a}(1 \pm 0.4,0,0)$. Two distinct gaps exist in the system: $\Delta_{Q^{*}} \approx 5 \mathrm{meV}$, which is related to the transport properties and $\Delta_{Q_{0}} \approx 2.5 \mathrm{meV}$, which characterizes the $\mathrm{HO}$ phase itself [21,22]. The $\Delta_{Q_{0}}$ gap only exists in the $\mathrm{HO}$ phase whereas the $\Delta_{Q^{*}}$ gap also exists in the AF phase [22].

\section{Numerical results}

The direct gap $\Delta_{X_{1}}$ (see in Appendix A's Introduction, Secs. $1 \mathrm{c}$ and $1 \mathrm{~d}$ ) only exists in the KMSL state and vanishes at the KMSL-AF to AF phase transition [see Fig. 6(a)]. Moreover, $\Delta_{X_{1}}$ is associated with the vector $Q$ (see in Appendix A Secs. $1 \mathrm{c}$ and $1 \mathrm{~d}$ ).

$\Delta_{X_{1}}$ vanishes continuously at the temperature $T_{0}=17,5 \mathrm{~K}$ [see Fig. 6(b)] confirming the second-order nature of the KMSL phase transition. The properties of the direct gap $\Delta_{X_{1}}$ are the same as that of the gap $\Delta_{Q_{0}}$ observed by INS and remain characteristic of the MSL phase. Note that these similarities have to be confirmed on the more realistic 3D structure.

\section{Evolution of the spectral density}

In this section, we analyze the local density of states $\rho(\omega)=\frac{-1}{2 N \pi} \sum_{k} \operatorname{Im}\left[G_{c}(\omega, k)\right]$ where the conduction electron Green's function $G_{c}(\omega, k)$ is computed from the mean-field Hamiltonian (5). From the spectral density, we will study the hybridization Kondo gap.

\section{Main experimental results}

Point Contact Spectroscopy (PCS) measurements exhibit a conductance gap opening at $T_{0}[26,98]$ which is characteristic of the $\mathrm{HO}$ phase. Above $T_{0}$, they show a resonance at the Fermi level appearing around $T \approx 70 \mathrm{k}$ and consistent with a hybridization Kondo gap [26]. Moreover, conductance 

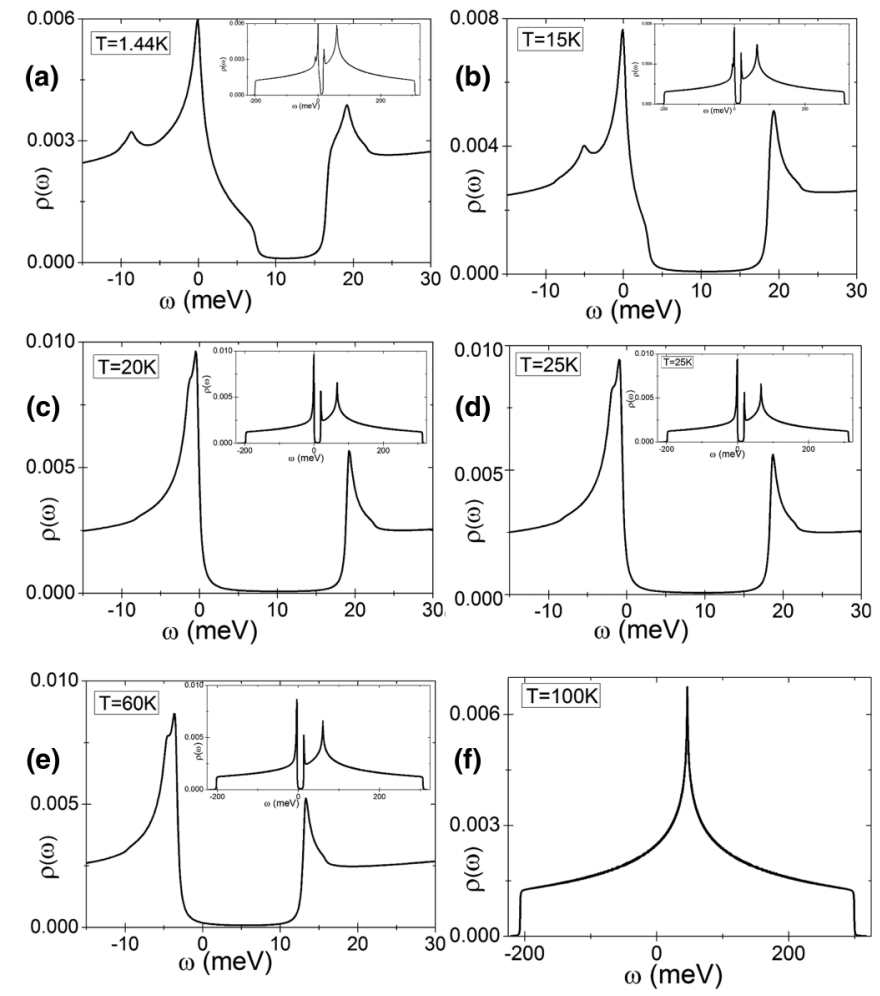

FIG. 7. Spectral density in the $\mathrm{URu}_{2} \mathrm{Si}_{2}$ for a KMSL ground state $\left(J_{\mathrm{SL}}=11.37 \mathrm{meV}\right.$ and $\left.J_{\mathrm{AF}}=0\right)$ in the effectively decoupled hightemperature phase (a) at $T=100 \mathrm{~K}$, the Kondo phase (b) at $T=60 \mathrm{~K}$, (c) at $T=25 \mathrm{~K}$, and (d) at $T=20 \mathrm{~K}$, and in the KMSL phase (e) at $T=15 \mathrm{~K}$ and (f) at $T=1.44 \mathrm{~K}$. The van Hove singularity appearing in figure (a) is standard for a tight-binding model on a square lattice, and it would coincide with the Fermi level only at electronic half-filling. Here, we precisely chose $n_{c}=0.7$ in order to locate the Fermi level sufficiently away from this singularity which has no physical meaning for $\mathrm{URu}_{2} \mathrm{Si}_{2}$.

measurements with STM show the formation of a Kondo-Fano resonance around $70 \mathrm{~K}$ related to a Kondo hybridization process [25]. These STM experiments clearly see the onset of another gap opening at $T_{0}$ related to the $\mathrm{HO}$ phase and independent of the hybridization Kondo gap [25]. Recently, optical conductivity experiments exhibit the emergence of a hybridization gap around $12 \mathrm{meV}$ at $T=30 \mathrm{~K}$, above $T_{0}$ and below the Kondo temperature $T_{K} \approx 70 \mathrm{~K}[31,32]$. This consideration has been reinforced by the ARPES observation of an electronic band close to the Fermi level [88]. Nevertheless, the precise origin of this indirect gap and its consequences on the HO phase are still under discussion [29-31].

\section{Numerical results}

The evolution of the spectral density for different temperatures is presented in Fig. 7. We see that a hybridization Kondo gap appears in the Kondo phase and increases its magnitude with the decrease of temperature [see Figs. 7(c) and 7(e)]. Below the KMSL phase transition, we see the appearance of an additional low-energy structure which is characteristic of the MSL order.

This hybridization Kondo gap remains independent of the MSL gap which appears below $T_{0}$ (see discussion in
Sec. IV C). The evolution and the temperature of appearance of the hybridization Kondo gap presented in Fig. 7 fits the evolution of the hybridization gap observed by PCS [26] and STM [25]. However, the spectral density does not explain the hybridization gap observed around $T=30 \mathrm{~K}$ by optical conductivity measurements [31,32].

We suggest that this observed hybridization gap could be related to the partial Kondo screening existing at $T=30 \mathrm{~K}$ since this temperature is in-between the Kondo temperature and the coherence temperature of the Fermi liquid (see discussion in Sec. IV A). Moreover, this hybridization gap observed at $T=30 \mathrm{~K}$ appears together with a maximum of the scattering rate [31]. As proposed in Ref. [31], this gap could be the signature of an interband process with a transition across the hybridization Kondo gap.

\section{v. CONCLUSION}

In this paper, we studied the stability of the MSL phase proposed earlier as a promising candidate for the $\mathrm{HO}$ phase of $\mathrm{URu}_{2} \mathrm{Si}_{2}$, with respect to the coupling to the charge degrees of freedom. In order to do so, we introduce a hybridization term originating from a Kondo interaction coupling the heavy electron bands to a light conduction band in the formalism. The charge degrees of freedom associated to the spinons have been treated via a slave-boson approach. Note that our model assumes the stability of a spin-liquid order parameter which is implemented phenomenologically. The effective existence of this spin-liquid solution needs deeper consideration of frustration and fluctuations effects in the realistic bct lattice as studied, for example, in Ref. [51].

We thus demonstrate the existence of a stable solution for the Kondo-MSL-AF phase diagram which generalizes the earlier approach $[49,50]$ and takes explicitly into account the charge fluctuations. The modulated spin liquid is a nonconventional ordered phase which, together with the AF magnetic order, competes with the Kondo screening. As a signature of our model, a $\mathrm{QCP}^{\star}$ and a $\mathrm{QCP}^{c}$ emerge from this competition of the Kondo effect and MSL formation. In particular, the KMSL phase, which displays the coexistence of Kondo and MSL order, is a new feature of this theory.

This stable solution produces two distinct gaps in the electronic band structure. One gap is related to the Kondo screening, the other is related to the MSL order parameter which is pinned at the commensurate vector $Q$. These two gaps can be considered independently and mark a clear distinction between the magnetic and the conducting properties of these systems.

Although we have performed a simple study on the square lattice, neglecting many of the complexities inherent to the band structure and the $5 f$ character of the $\mathrm{U}$ atom, our results are promising regarding the experimental phase diagram for $\mathrm{URu}_{2} \mathrm{Si}_{2}[19]$.

We found a first-order KMSL to AF phase transition and a second-order Kondo to KMSL phase transition. Note that the order of the transition will have to be confirmed by fluctuation effects calculations, left for later work. A KMSL$\mathrm{AF}$ coexisting phase appears in the numerical phase diagram (see Fig. 4) between the KMSL and AF phase. This phase diagram will have to be studied considering a more realistic 
approach to see whether this coexisting phase disappears or not.

Furthermore, we find a Fermi surface reconstruction at the KSL/KMSL phase transition as observed in several experiments [28,88-90]. We also find that the Fermi surfaces between the KMSL and the AF phases are similar to each other as denoted in Ref. [97].

We also find a variation of entropy around $0.3 k_{B} \ln (2)$ which is close to the value measured in the realistic compound [12]. Here, the entropy freezing is a two-step scenario with a partial Kondo screening at $T_{K}$ similar to the one described in Ref. [63]. The remaining entropy is then frozen in by the MSL ordering.

Our model provides results in agreement with the temperature evolution of the entropy, the Sommerfeld coefficient, and the spectral density in $\mathrm{URu}_{2} \mathrm{Si}_{2}$. Our results are also in qualitative agreement with the evolution of the Fermi surface, the MSL gap evolution, and the phase diagram. The extension of our work to the realistic 3D lattice should produce further quantitative agreement in the (T,P) phase diagram and in the gap and Fermi surface evolutions. Observe that in order to achieve a more quantitative agreement, it will be necessary to consider the local structure of the $U$ orbital which has multipolar aspects and an Ising-type anisotropy. Finally, we can also envisage that it should be possible to calculate realistic electronic band structure to establish more direct contact with ARPES, Raman scattering, and inelastic neutron scattering experiments.

\section{ACKNOWLEDGMENTS}

The authors thanks M.-A. Méasson for helpful discussion. This research was carried out with the aid of the Computer System of High Performance of the International Institute of Physics UFRN, Natal, Brazil. This work was also supported, in part, by the European IRSES program SIMTECH (Contract No. 246937), by CAPES-COFECUB (Brazil-France), and by CNPq (Brazil).

\section{APPENDIX A: ELECTRONIC BAND STRUCTURE}

\section{General considerations}

The phase diagram presented in Sec. III reveals the existence of several phases characterized by different order parameters. In the present section, we exhibit the specific signature of each of these phases on the electronic band structure and on their associated Fermi surfaces.

We present the electronic band structure in the first Brillouin zone of the square lattice (FBZ) (see Fig. 8). The electronic band dispersion is plotted along the four directions $M^{\prime} \Gamma-$ $\Gamma X-X M-M \Gamma$ (see Fig. 8) where $M^{\prime}, \Gamma, X$, and $M$ are the high-symmetry points of the FBZ. Note that the direction $M^{\prime} \Gamma$ is considered here to emphasize the $Z 4$ symmetry breaking in the MSL phases.

The zone folding manifesting itself in the AF and MSL phases reduces the first Brillouin zone to the magnetic Brillouin zone (MBZ) (dotted line in Fig. 8). The boundaries of the $\mathrm{MBZ}$ are delimited in the four directions $k_{x}= \pm k_{y} \pm \pi$. For simplicity, we signalized the two characteristic points localized on the bound of the MBZ: $X_{1}$ in $\left(k_{x}=\pi / 2, k_{y}=\pi / 2\right)$ and $X_{2}$ in $\left(k_{x}=-\pi / 2, k_{y}=\pi / 2\right)$.

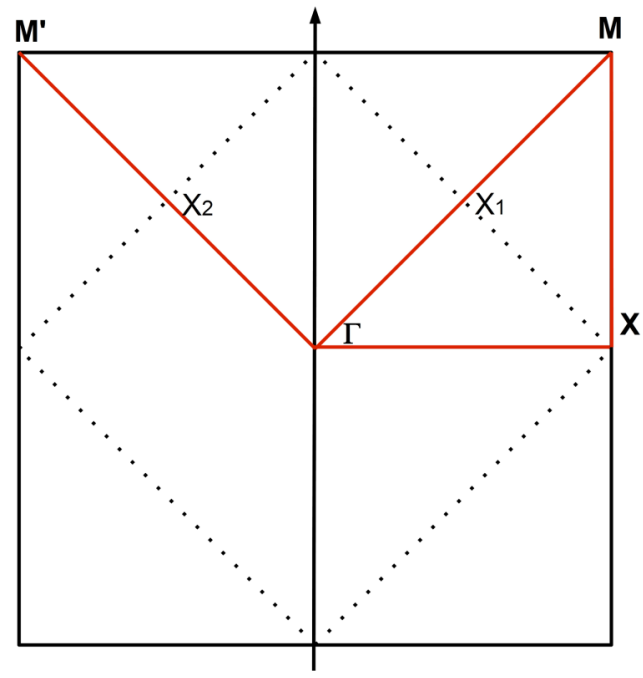

FIG. 8. (Color online) The first Brillouin zone of the square lattice is presented by the solid line. For simplicity, the side of the real square lattice is set to $a=1$. The Brillouin zone used in the case of folded Fermi surface is represented by the dotted line. The high-symmetry point of the first Brillouin zone $\Gamma$ is the center of the zone, the center of a side $X$, and a corner $M\left(\right.$ or $\left.M^{\prime}\right)$. The characteristic direction of the first Brillouin zone is given by the straight lines $\Gamma M$, $\Gamma X, X M$, and $\Gamma M^{\prime}$. The $X_{1}$ and $X_{2}$ are two characteristic points of the magnetic Brillouin zone.

For each dispersion, we plot the original and the folded electronic band dispersions. The original and folded electronic dispersions are related to the wave vector $Q$. Consequently, an excitation between these two layers indicates the presence of folding and may be related to the excitation wave vector $Q$.

\section{Electronic dispersion in the different phases}

\section{a. Disordered phases}

The decoupled phase: Paramagnetic metal and free moments. The electronic band structure of the metal + free moments phase is presented in Fig. 9. The spinons are degenerate, dispersionless, and centered at the zero-energy level. The spinons are noninteracting and nondispersive. Note that the energy level of the band crossing [solid and dashed lines in Fig. 9(c)] occurring at the $X, X_{1}$, and $X_{2}$ points is determined by the chemical potential $\mu_{c}$. These band crossings between the layer and the folded layer occur in the four directions $k_{y}= \pm \pi \pm k_{x}$. This helps to determine the boundaries of the reduced Brillouin zone (dashed line in Fig. 8). Nevertheless, the conduction electron dispersion does not present any folding signature, as expected for the metallic phase.

The Kondo phase with $\phi_{0}=0$. The electronic band structure and the electronic dispersion of the Kondo phase with $\phi_{0}=$ 0 are plotted in Fig. 10. There is now the appearance of a gap in the electronic band structure around the Fermi level. The hybridization between the conduction electrons and the local moments leads to an extended Fermi surface (FS). This FS enlargement is associated with the presence of the Kondo quasiparticles in the system and is related to the decreasing of 
(a)
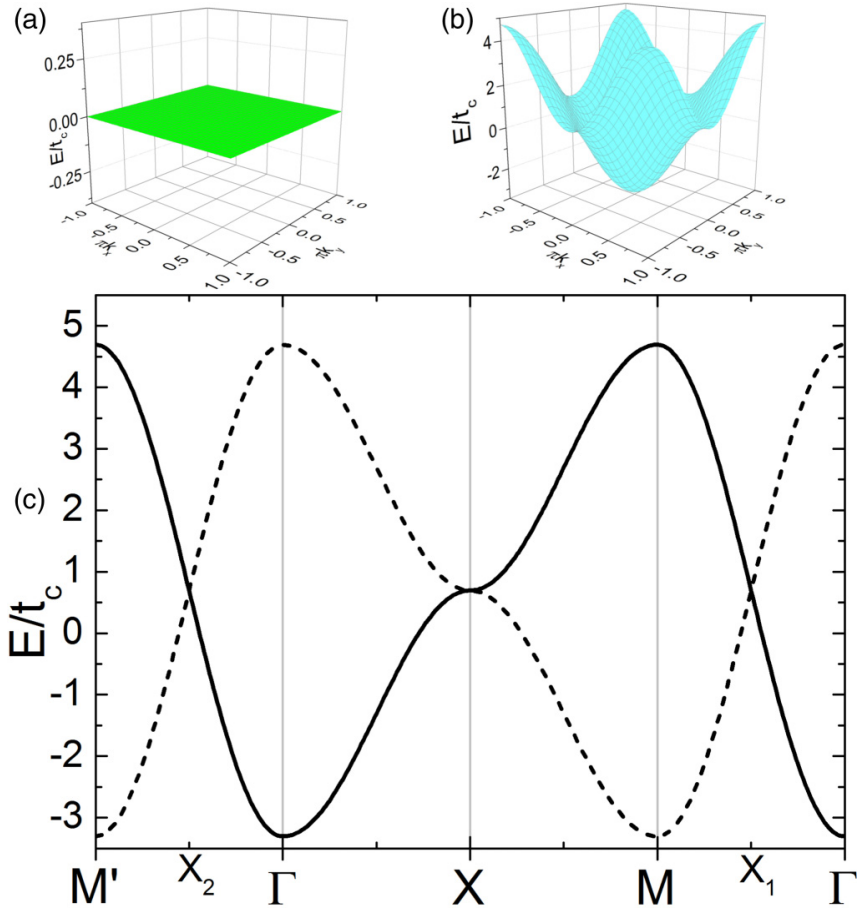

FIG. 9. (Color online) The electronic band structure of the local moments (a) and the conduction electrons (b) in the paramagnetic metal + free moments phase in the first Brillouin zone. In this case $\phi_{0}=0, \phi_{Q}=0, \rho=0, \theta=0, S_{Q}=0, \mu=0$, and $\mu_{c} / T_{K 0}=-7.45$ at $T / T_{K 0}=2, J_{\mathrm{SL}}=0$, and $J_{\mathrm{AF}}=0$. In figure (c) are plotted the original (solid line) and folded (dashed line) dispersions of the $c$ electron in the characteristic directions of the first Brillouin zone. The Fermi level is set at $E=0$.

the resistivity occurring below $T_{K}$ in Kondo lattice systems [1]. This indirect gap induced by Kondo interaction [see Fig. 10 (b)] is approximatively equal to $\left.\frac{1}{2}\left[t_{c}-\sqrt{(} t_{c}^{2}+4 \rho^{2}\right)\right]$. It depends on the electronic bandwidth $t_{c}$ and on the amplitude of the Kondo coupling $\rho$ and it vanishes together with the Kondo phases.

The Kondo phase with $\phi_{0} \neq 0$. The electronic band structure and the single-particle dispersion of the Kondo phase with $\phi_{0} \neq 0$ are plotted on Fig. 11. In this phase, the Kondo mechanism occurs between two layers with opposite dispersions. The main feature is a direct gap opening between the two layers in contrast with the indirect gap opening in the classical Kondo phase. The direct gap at the $\Gamma$ point is proportional to $\rho^{2} / 4 t_{c}$. This gap vanishes with the Kondo phase. Note that no symmetry is broken between the $\phi=0$ and $\phi \neq 0$ Kondo phases. This is typical of a crossover separating these two phases. This crossover is also accompanied by a change of the Fermi surface [see Figs. 5(d) and 5(e)].

\section{b. Decoupled phase: $A F+$ metal}

In the AF phase, the electronic band structure is composed by the local moments [see Fig. 12(a)] and the conduction electrons [see Fig. 12(b)]. In our model, the spinons in the AF phase are totally flat and nondegenerate [see Fig. 12(a)]. The gap between the two spinons is equal to $8 S_{Q}$.

From the magnetic-type Kondo mean-field decoupling [56], we deduce the magnetic interaction of the
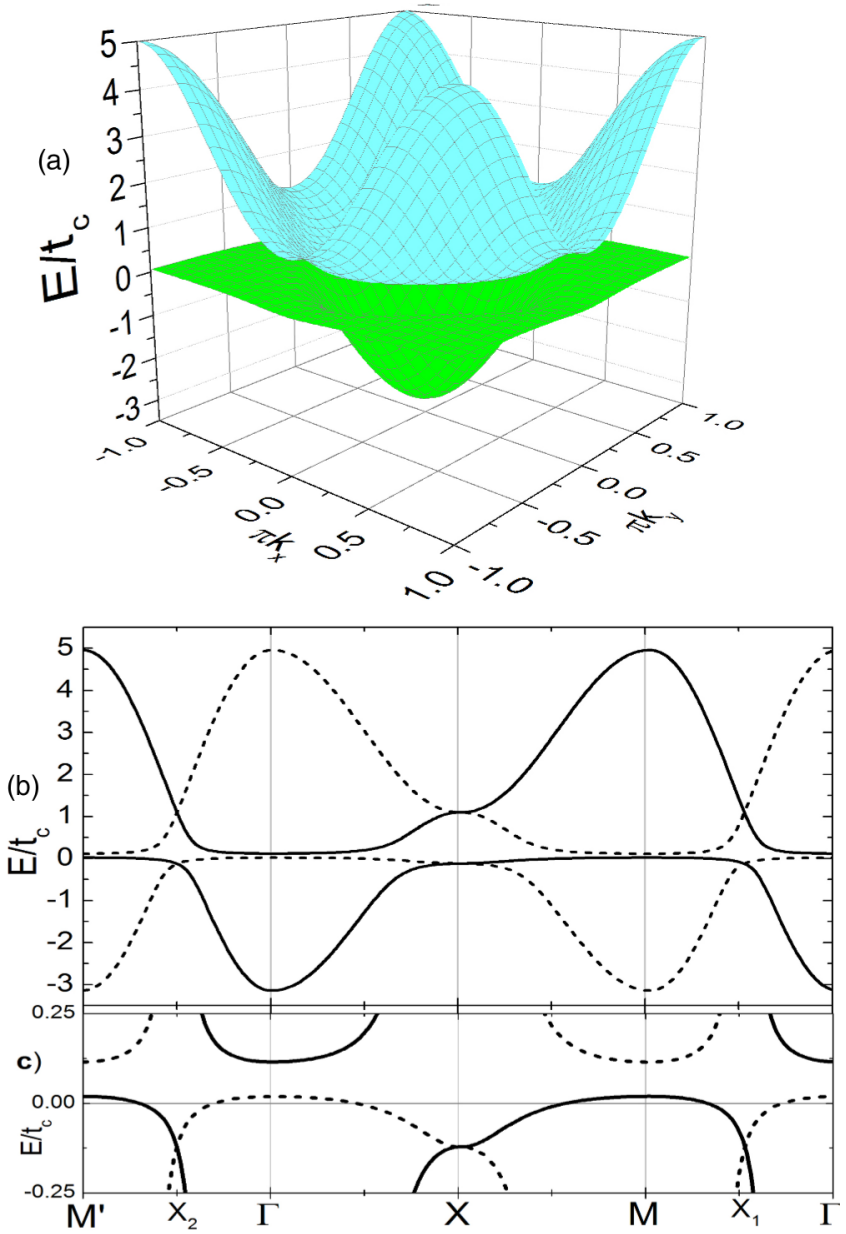

FIG. 10. (Color online) In (a), the electronic band structure of the Kondo phase with $\phi_{0}=0$ in the first Brillouin zone. In this case $\phi_{0}=\phi_{Q}=0, \rho / T_{K 0}=4.40, \theta=0, S_{Q}=0, \mu / T_{K 0}=-0.58$, and $\mu_{c} / T_{K 0}=-9.37$ with $T / T_{K 0}=0.5, J_{\mathrm{SL}}=0$, and $J_{\mathrm{AF}}=0$. In figure (b) is plotted the original (solid line) and folded (dashed line) dispersions of the electronic band in the four characteristic directions of the first Brillouin zone. The zoom near the Fermi level $(E=0)$ is shown in the figure (c). Note the direct gap in the $\Gamma$ and $M$ points.

local moments with the conduction electrons. Considering the first-order perturbation of the Kondo interaction in the AF phase, the mean-field Kondo lattice Hamiltonian writes $H_{\mathrm{KL}}^{\mathrm{MF}} \approx J_{K} \sum_{q, \sigma}\left[c_{q \sigma}^{+}\left(\sigma S_{Q}\right) c_{q-Q \sigma}\right]$. The conducting electron spectrum writes $E^{c} \approx \frac{1}{2} t_{c}\left(\epsilon_{q}+\epsilon_{q-Q}\right)-\lambda \pm$ $\frac{1}{2} \sqrt{t_{c}^{2}\left(\epsilon_{q-Q}-\epsilon_{q}\right)^{2}+4 S_{Q}}$. The magnetization of the local moments induces a gap in the conduction electrons band structure [see Figs. 12(c) and 12(d)]. The direct gap at the $X, X_{1}$, and $X_{2}$ points between the original (solid line) and the folded (dashed line) layer equals $2 S_{Q}$ (see Fig. 12). The magnetization seen by the conduction electrons is four times smaller than the magnetization for the corresponding local moments because of the local aspect of the Kondo coupling. This gap opening is accompanied by a folding of the conduction electron band structure in relation with the lattice symmetry breaking. Moreover, this gap is related to the commensurable vector $Q$. 

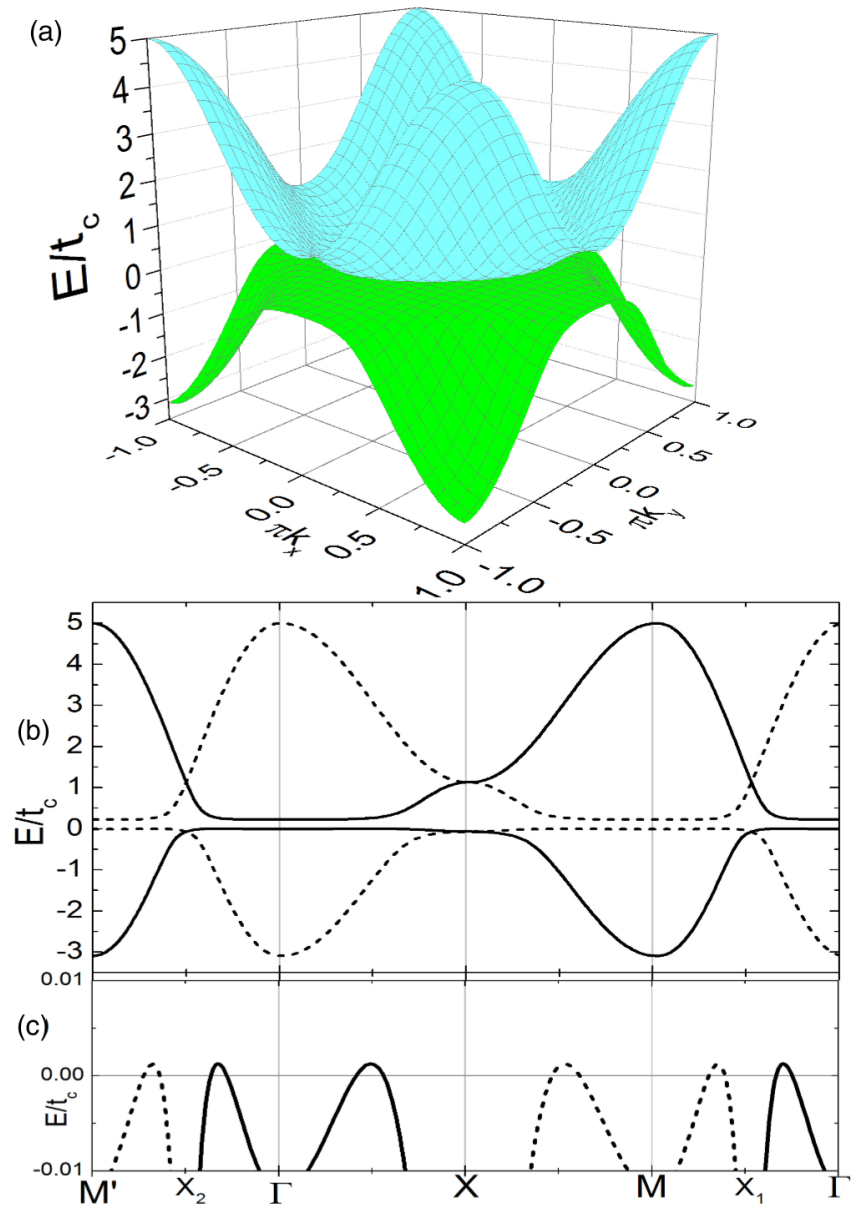

FIG. 11. (Color online) In (a), the electronic band structure of the Kondo phase $\phi_{0} \neq 0$ in the first Brillouin zone. In this case $\phi_{0} / T_{K 0}=$ $0.19, \phi_{Q}=0, \rho / T_{K 0}=4.25, \theta=-\pi / 2, S_{Q}=0, \mu / T_{K 0}=-0.99$, and $\mu_{c} / T_{K 0}=-9.81$ with $T / T_{K 0}=0.5, J_{\mathrm{SL}} / T_{K 0}=0.5$, and $J_{\mathrm{AF}}=$ 0 . In figure (b) we plot the original (solid line) and folded (dashed line) dispersions of the electronic band in the four characteristic directions of the first Brillouin zone. The zoom near the Fermi level $(E=0)$ is shown in figure (c). Note the direct gap in the $\Gamma$ and $M$ points.

\section{c. MSL phases}

The decoupled phase: $M S L+$ metal. The MSL phase is an itinerant phase associated with the dispersion relation $\gamma_{q-\frac{Q}{2}}$ whose dispersion is presented in Fig. 13 . We clearly see a $Z^{2} 4$ symmetry breaking with the opening of the direct gap at the point $X_{1}$, called $\Delta_{X_{1}}$, which is absent at the $X_{2}$ point [see Fig. 13(c)]. This $Z 4$ symmetry breaking has been introduced in the initial MSL model [49] following the observation on susceptibility measurements [72] inside the HO phase of $\mathrm{URu}_{2} \mathrm{Si}_{2}$ compound. At the $X_{2}$ point, the two bands are in contact with the Fermi level. In fact, this contact exists along the flat bands directions $k_{y}= \pm \pi+k_{x}$. Note that either hole or electron pockets emerge along these two directions in the spinon dispersion at the conduction electron Fermi surface in the presence of the second-nearest-neighbor term in the dispersion relation [49]. The gap at the $X_{1}$ point $\Delta_{X_{1}}=8 \phi_{Q}$ can be associated with the vector $Q$ and depends only on the modulated spin-liquid mean-field parameters The spinon dispersion reveals a nonsymmetrical folding, originating in the

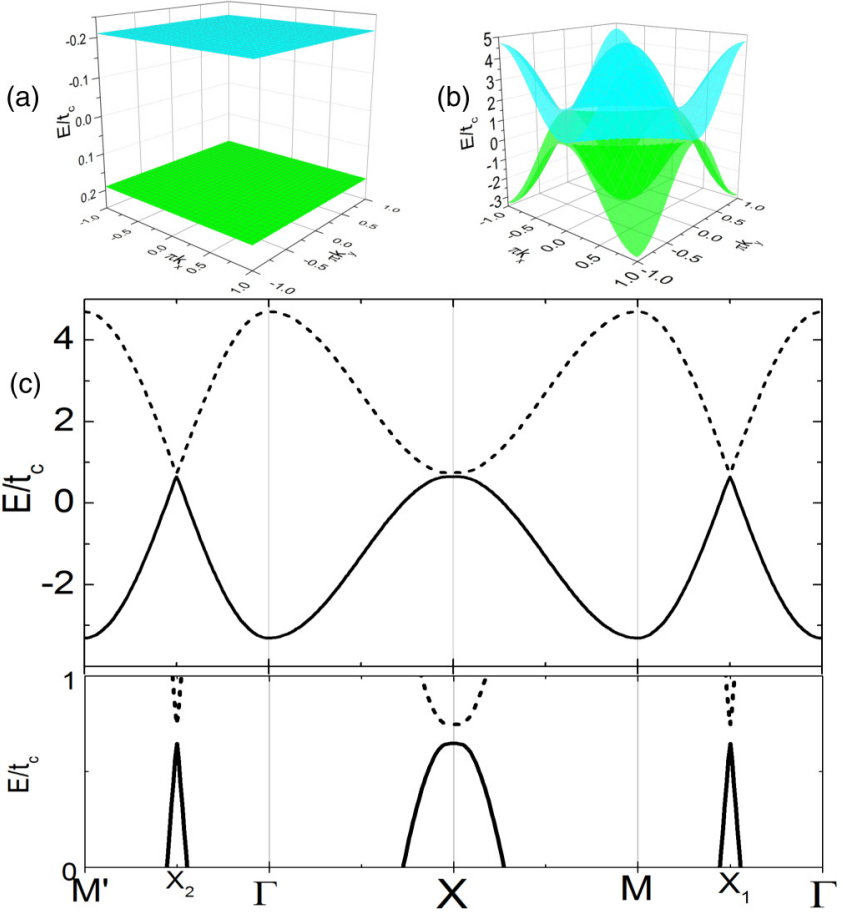

FIG. 12. (Color online) In (a), the electronic band structure of the the $f$ electrons and the conducting electrons (b) in the AF phase in the first Brillouin zone. In the AF state $\phi_{0}=0, \phi_{Q}=0, \rho=0, \theta=0$, $S_{Q} / T_{K 0}=0.51, \mu / T_{K 0}=0.10$, and $\mu_{c}=-7.10$ at $T / T_{K 0}=0.01$, $J_{\mathrm{SL}}=0$. and $J_{\mathrm{AF}} / T_{K 0}=0.5$. In figure (c) are plotted the original (solid line) and the folded (dashed line) dispersions of the $c$ electron band in the characteristic directions of the first Brillouin zone. The zoom near the Fermi level $(E=0)$ is shown in figure (d).

association of Néel order lattice symmetry breaking with Z4 symmetry breaking.

The correlated phase: KMSL. The transformation of the electronic band structure at the Kondo-KMSL phase transition can be observed throughout Figs. 11 and 14. We observe a strong electronic band reconstruction with the emergence of folding due to the lattice symmetry breaking. Moreover, the opening of a gap only at the $X_{1}$ point emphasizes the $Z 4$ symmetry breaking in the KMSL phase. This gap is also associated with the commensurate vector $Q$. The $Z 4$ symmetry breaking is related to the modulated spin-liquid mean field $\phi_{Q}$. The direct gap occurring at the $X_{1}$ point $\Delta_{X_{1}}$ is approximatively equal to $4 \phi_{Q}$. This gap is a characteristic signature of the MSL phase.

\section{d. Coexisting phase}

The dispersion of the electronic band structure of the coexisting KMSL-AF state is displayed in Fig. 15. The gap induced by the modulated spin liquid is compensated by the AF gap. Consequently, the Z4 symmetry breaking is progressively destroyed. On the electronic band dispersion shown in Fig. 15(c), we see the onset of a gap between the original and folded electronic bands that does not exist in the pure KMSL phase electronic dispersion [see Fig. 14(c)]. This direct gap at the $X_{2}$ and the $\mathrm{X}$ points $\left(k_{x}=\pi, k_{y}=0\right)$ is approximately equal to $4 S_{Q}$. It is directly related to the 

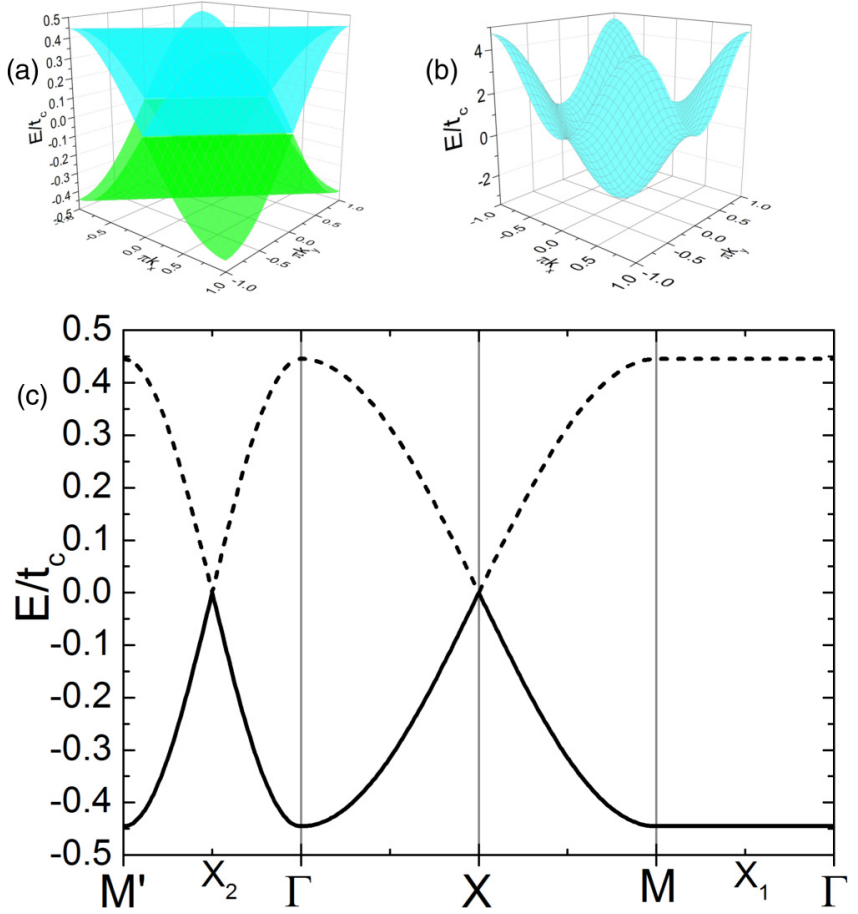

FIG. 13. (Color online) In (a), the electronic band structure of the the $f$ electrons and the conducting electrons (b) in the MSL phase in the first Brillouin zone. In the MSL state $\phi_{0} / T_{K 0}=1.14$, $\phi_{Q} / T_{K 0}=1.14, \rho=0, \theta=0, S_{Q}=0, \mu=0$, and $\mu_{c} / T_{K 0}=-7.10$ with $T / T_{K 0}=0.01, J_{\mathrm{SL}} / T_{K 0}=5$, and $J_{\mathrm{AF}}=0$. In figure (c) are plotted the original (solid line) and folded (dashed line) dispersions of the $c$ electron band in the characteristic directions of the first Brillouin zone. The Fermi level is set at $E=0$.

magnetization of the system. This gap exists only in the antiferromagnetic phase and implies a less pronounced aspect of the $Z 4$ symmetry of the Fermi surface. The direct gap $\Delta_{X_{1}}$ is approximately equal to $4 \sqrt{\phi_{O}^{2}+S_{O}^{2}}$ and also depends on the magnetization of the system. Note that both direct gaps $\Delta_{X_{1}}$ and $\Delta_{X_{2}}$ are related to the wave vector $Q$ and this emphasizes the similar symmetry breaking between the AF and MSL phases.

\section{APPENDIX B: FERMI SURFACES: CHARACTERISTIC SIGNATURES OF THE DIFFERENT PHASES}

We display the evolution of the electronic band structure between the different phases. We observe the onset of gaps the systems characterizing the existing different phases. Moreover, we see that the electronic band structure evolves in the different phases, as shown in Fig. 3. The system can exhibit four types of coexisting order parameters.

\section{Kondo order parameter}

The Kondo effect is characterized by a volume change of the Fermi surface. This change of volume is a consequence of the hybridization of the $f$ and $c$ electrons. It occurs between the decoupled phases [see Figs. 5(a), 5(b), and 5(f)] and the Kondo phase [Figs. 5 d) and 5(e)]. This explains the Kondo breakdown and the accompanied existence of the QCP in the phase
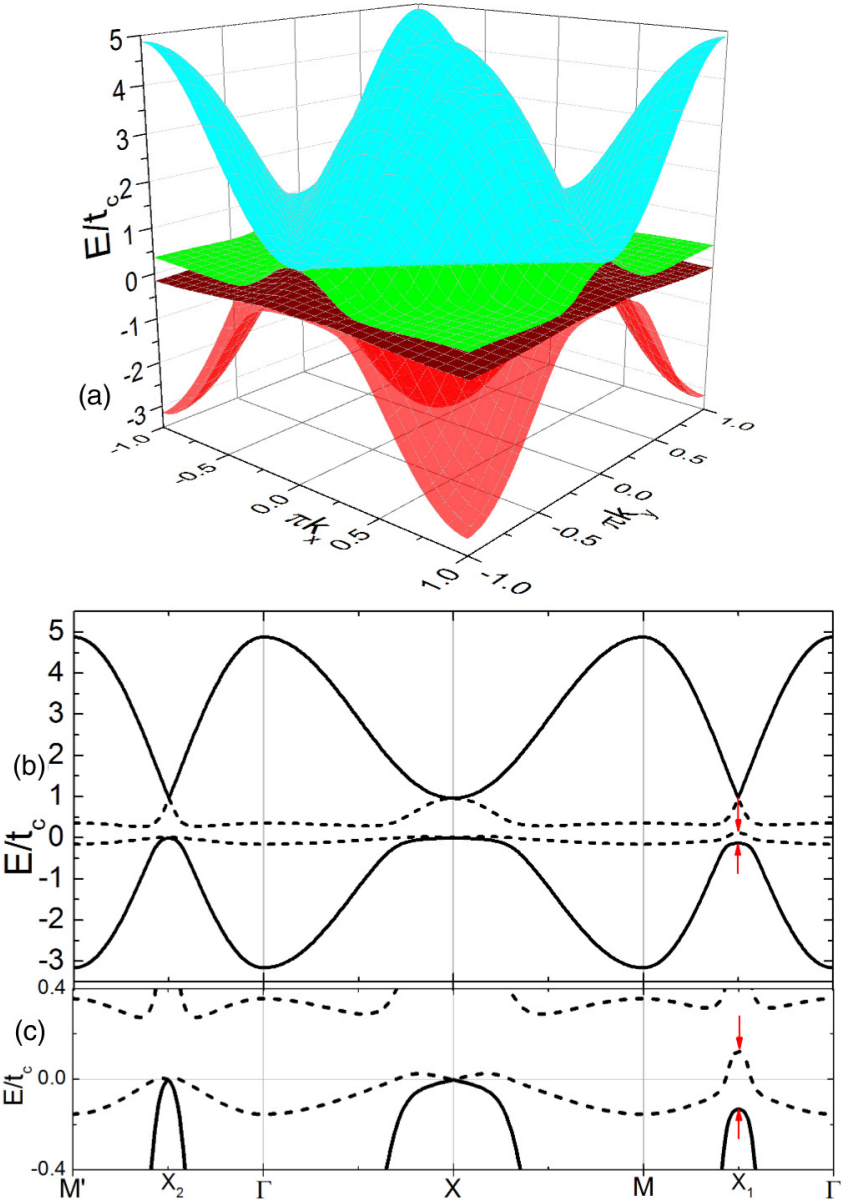

FIG. 14. (Color online) In (a), the electronic band structure of the KMSL phase in the first Brillouin zone. In this case $\phi_{0} / T_{K 0}=$ $0.6, \phi_{Q} / T_{K 0}=0.36, \rho / T_{K 0}=2.99, \theta=-\pi / 2, S_{Q}=0, \mu / T_{K 0}=$ -0.98 , and $\mu_{c} / T_{K 0}=-8.8$ with $T / T_{K 0}=0.01, J_{\mathrm{SL}} / T_{K 0}=1.84$, and $J_{\mathrm{AF}} / T_{K 0}=0.05$. In figure (b) are plotted the original (solid line) and folded (dashed line) dispersions of the electronic band in the four characteristic directions of the first Brillouin zone. The zoom near the Fermi level $(E=0)$ is shown in the figure (c). Note the direct gap in the $\Gamma$ and $M$ points and the gap at the $X_{1}$ point.

diagram at $J_{\mathrm{SL}}^{\star}$ and $J_{\mathrm{AF}}^{\star c}$ (see Fig. 1). The Kondo breakdown is difficult to observe at the KMSL to AF phase transition due to the lattice symmetry breaking occurring simultaneously at this phase transition. Nevertheless, the change of Fermi surface volume, without folding, is a clear signature of the Kondo effect.

\section{Lattice symmetry breaking}

The lattice symmetry breaking occurring in the magnetic ordered phases manifests itself in the folding (or nonsymmetrical folding) of the Fermi surface [see Figs. 5(b), 5(c), 5(f), and $5(\mathrm{~g})]$. This lattice symmetry breaking is a clear signature of the magnetically ordered nature of these phases. The folding is observable by the periodicity occurring in the Fermi surface. We see that this periodicity is related to the limit of the MBZ. This folding can also be observed by neutron scattering. The folding of the Fermi surface is a typical feature of 

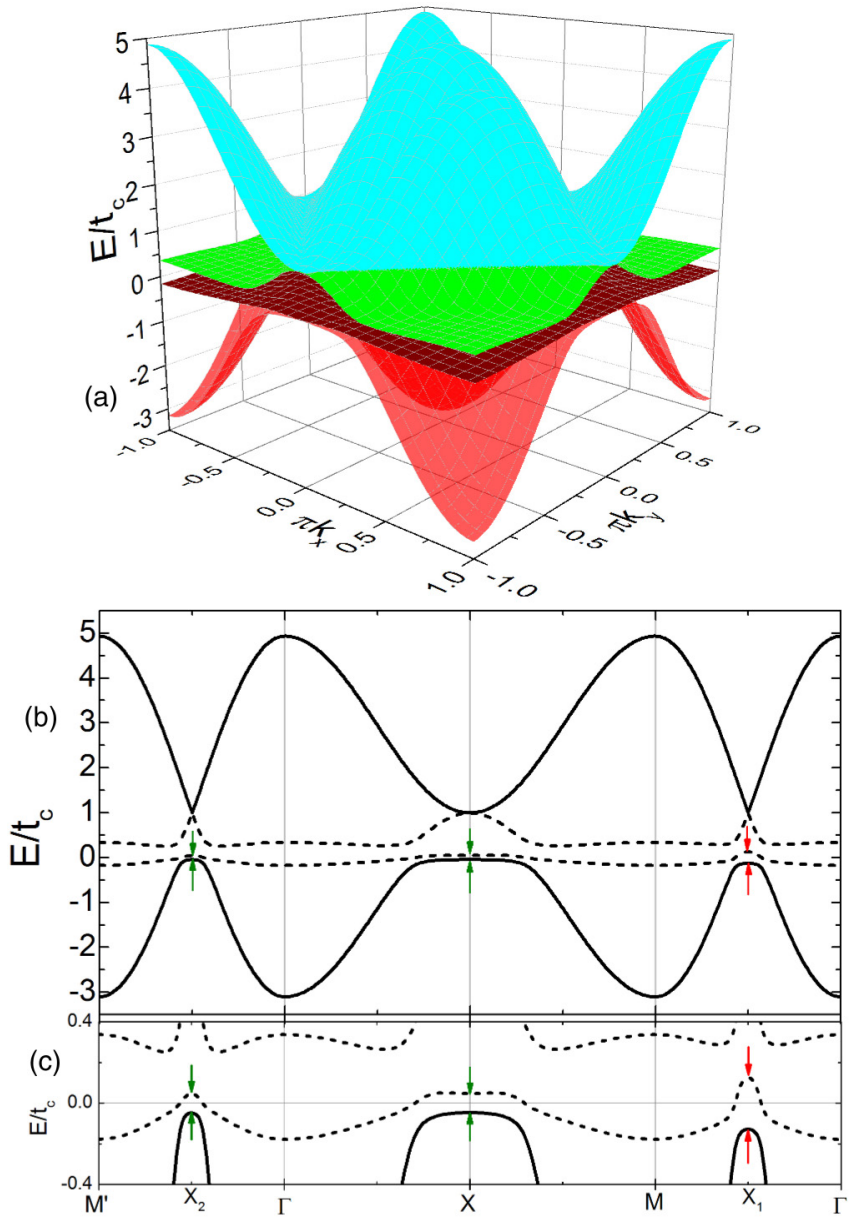

FIG. 15. (Color online) In (a), the electronic band structure of the KMSL-AF phase in the first Brillouin zone. In this case $\phi_{0} / T_{K 0}=0.6, \phi_{Q} / T_{K 0}=0.33, \rho / T_{K 0}=2.7, \quad \theta=-\pi / 2$, $S_{Q} / T_{K 0}=0.13, \mu / T_{K 0}=-0.78$, and $\mu_{c} / T_{K 0}=-8,35$ with $T / T_{K 0}=0.01, J_{\mathrm{SL}} / T_{K 0}=1.84$, and $J_{\mathrm{AF}} / T_{K 0}=0.15$. In figure (b) are plotted the original (solid line) and folded (dashed line) dispersions of the electronic band in the four characteristic directions of the first Brillouin zone. The zoom near the Fermi level $(E=0)$ is shown in the figure (c). magnetically ordered phases (AF and MSL), thus, resulting in the enlargement of the Fermi surface which, in this case, is not related to Kondo breakdown physics.

\section{Z4 symmetry breaking}

This symmetry is broken in the Fermi surfaces with MSL charateristics [see Figs. 5(c), 5(f), and 5(g)]. The Z4 symmetry breaking is emphasized by the direct gap opening in the electronic dispersion along the flat band direction $k_{y}=-k_{x} \pm \pi$ which is observable at $X_{1}$ on Figs. 13, 14, and 15 . The $Z 4$ symmetry breaking of the Fermi surface is a characteristic signature of the MSL states.

\section{Time-reversal symmetry breaking}

The time-reversal symmetry occurs only in the AF phase and originates the AF order parameter. This symmetry cannot be directly seen on the Fermi surface. However, the electronic dispersion associated with the AF phase (see Figs. 12 and 15) exhibits a gap opening between the two conduction electron bands. This specific gap, proportional to $S_{Q}$ and related to the commensurate vector $Q$, could be a specific signature of the time-reversal symmetry.

The difference between the Kondo phases with $\phi_{0}=0$ and $\phi_{0} \neq 0$ is observable in the electronic band structure and in the corresponding Fermi surfaces. If the Fermi surfaces of these two phases are different from each other, they preserve the same symmetry and conserve the same volume. A crossover could describe the transition between these two phases. A gauge invariance manifests itself in the Hamiltonian. We see that the Hamiltonian is invariant under the transformation $\left(\phi_{0}, \phi_{Q}, b_{0}, i b_{Q}\right) \longrightarrow\left(-\phi_{0},-\phi_{Q}, i b_{Q}, b_{0}\right)$. This kind of invariance corresponds to the changing of phases of the $f$ fermions such as $f_{R, \sigma} \longrightarrow e^{i\left(\frac{\pi}{2}-Q \cdot R\right)} f_{R, \sigma}$. This invariance also explains the existence of the Kondo phase with $\phi_{0} \neq 0$. The inhomogeneous coupling between the two phases can be understood as a homogeneous Kondo coupling between two layers with opposite dispersions. These homogeneous couplings do not change the symmetry of the phases.
[1] A. C. Hewson, The Kondo Problem to Heavy Fermions. (Cambridge University Press, Cambridge, England, 1993).

[2] P. Coleman, C. Pépin, Q. Si, and R. Ramazashvili, J. Phys.: Condens. Matter 13, R723 (2001).

[3] G. R. Stewart, Rev. Mod. Phys. 73, 797 (2001).

[4] H. V. Löhneysen, A. Rosch, M. Vojta, and P. Wölfe, Rev. Mod. Phys. 79, 1015 (2007).

[5] F. Steglich, J. Aarts, C. D. Bredl, W. Lieke, D. Meschede, W. Franz, and H. Schafer, Phys. Rev. Lett. 43, 1892 (1979).

[6] S. Sachdev, Quantum Phase Transitions (Cambridge University Press, Cambridge, UK, 1999).

[7] Q. Si, S. Rabello, K. Ingersent, and J. L. Smith, Nature(London) 413, 804 (2001).

[8] S. Doniach, in Valence Instabilities and Related Narrow Band Phenomena, edited by R. D. Parks (Plenum, New York, 1977), p. 169; S. Doniach, Physica (Amsterdam) B 91, 231 (1977).
[9] J. A. Hertz, Phys. Rev. B 14, 1165 (1976).

[10] A. J. Millis, Phys. Rev. B 48, 7183 (1993).

[11] T. Moriya, Spin Fluctuations in Itinerant Electron Magnetism (Springer, Berlin, 1985).

[12] T. T. M. Palstra, A. A. Menovsky, J. van den Berg, A. J. Dirkmaat, P. H. Kes, G. J. Nieuwenhuys, and J. A. Mydosh, Phys. Rev. Lett. 55, 2727 (1985).

[13] U. Walter, C. K. Loong, M. Loewenhaupt, and W. Schlabitz, Phys. Rev. B 33, 7875 (1986).

[14] M. B. Maple, J. W. Chen, Y. Dalichaouch, T. Kohara, C. Rossel, M. S. Torikachvili, M. W. McElfresh, and J. D. Thompson, Phys. Rev. Lett. 56, 185 (1986).

[15] C. Broholm, J. K. Kjems, W. J. L. Buyers, P. Matthews, T. T. M. Palstra, A. A. Menovsky, and J. A. Mydosh, Phys. Rev. Lett. 58, 1467 (1987).

[16] A magnetization exists, however, it is too weak to characterize an AF order. 
[17] E. D. Isaacs, D. B. McWhan, R. N. Kleiman, D. J. Bishop, G. E. Ice, P. Zschack, B. D. Gaulin, T. E. Mason, J. D. Garrett, and W. J. L. Buyers, Phys. Rev. Lett. 65, 3185 (1990).

[18] J. A. Mydosh and P. M. Oppeneer, Rev. Mod. Phys. 83, 1301 (2011).

[19] A. Villaume, F. Bourdarot, E. Hassinger, S. Raymond, V. Taufour, D. Aoki, and J. Flouquet, Phys. Rev. B 78, 012504 (2008).

[20] F. Bourdarot, N. Martin, S. Raymond, L. P. Regnault, D. Aoki, V. Taufour, and J. Flouquet, Phys. Rev. B 84, 184430 (2011).

[21] C. R. Wiebe, J. A. Janik, G. J. MacDougall, G. M. Luke, J. D. Garrett, H. D. Zhou, Y.-J. Jo, L. Balicas, Y. Qiu, J. R. D. Copley, Z. Yamani, and W. J. L. Buyers, Nat. Phys. 3, 96 (2007).

[22] F. Bourdarot, E. Hassinger, S. Raymond, D. Aoki, V. Taufour, and J. Flouquet, J. Phys. Soc. Jpn. 79, 064719 (2010).

[23] E. Hassinger, G. Knebel, K. Izawa, P. Lejay, B. Salce, and J. Flouquet, Phys. Rev. B 77, 115117 (2008).

[24] M. M. Altarawneh, N. Harrison, S. E. Sebastian, L. Balicas, P. H. Tobash, J. D. Thompson, F. Ronning, and E. D. Bauer, Phys. Rev. Lett. 106, 146403 (2011).

[25] P. Aynajian, E. H. da Silva Neto, C. V. Parker, Y. Huang, A. Pasupathy, J. Mydosh, and Ali Yazdani, Proc. Natl. Acad. Sci. USA 107, 10383 (2010); A. R. Schmidt, M. H. Hamidian, P. Wahl, F. Meier, A. V. Balatsky, J. D. Garrett, T. J. Williams, G. M. Luke, and J. C. Davis, Nature (London) 465, 570 (2010).

[26] J. G. Rodrigo, F. Guinea, S. Vieira, and F. G. Aliev, Phys. Rev. B 55, 14318 (1997).

[27] J. D. Dellinger, G.-H. Gweon, J. W. Allen, C. G. Olson, Y. Dalichaouch, B.-W. Lee, M. B. Maple, Z. Fisk, P. C. Canfield, and P. E. Armstrong, Phys. B (Amsterdam) 281-282, 716 (2000); J. D. Dellinger, G.-H. Gweon, J. W. Allen, C. G. Olson, M. B. Maple, J. L. Sarrao, P. E. Armstrong, Z. Fisk, and H. Yamagami, J. Electron. Spectrosc. Relat. Phenom. 117-118, 347 (2001).

[28] A. Santander-Syro, M. Klein, Florin L. Boariu, A. Nuber, P. Lejay, and F. Reinert, Nat. Phys. 5, 637 (2009).

[29] U. Nagel, T. Uleksin, T. Rõõm, R. P. S. M. Lobo, P. Lejay, C. C. Homes, J. S. Hall, A. W. Kinross, S. K. Purdy, T. Munsie, T. J. Williams, G. M. Luke, and Thomas Timusk, Proc. Natl. Acad. Sci. USA 109, 19161 (2012).

[30] D. A. Bonn, J. D. Garrett, and T. Timusk, Phys. Rev. Lett. 61, 1305 (1988).

[31] J. Levallois, F. Lévy-Bertrand, M. K. Tran, D. Stricker, J. A. Mydosh, Y.-K. Huang, and D. van der Marel, Phys. Rev. B 84, 184420 (2011).

[32] W. K. Park, P. H. Tobash, F. Ronning, E. D. Bauer, J. L. Sarrao, J. D. Thompson, and L. H. Greene, Phys. Rev. Lett. 108, 246403 (2012).

[33] P. Fazekas, A. Kiss, and K. Radonczi, Prog. Theor. Phys. Suppl. 160, 114 (2005).

[34] F. Cricchio, F. Bultmark, O. Granas, and L. Nordstrom, Phys. Rev. Lett. 103, 107202 (2009).

[35] H. Kusunose and Y. Ohashi, J. Phys. Soc. Jpn. 80, 084702 (2011).

[36] A. I. Toth and G. Kotliar, Phys. Rev. Lett. 107, 266405 (2011).

[37] K. Haule and G. Kotliar, Europhys. Lett. 89, 57006 (2010).

[38] H. Ikeda and Y. Ohashi, Phys. Rev. Lett. 81, 3723 (1998).

[39] V. P. Mineev and M. E. Zhitomirsky, Phys. Rev. B 72, 014432 (2005).
[40] S. Elgazzar, J. Rusz, M. Amft, P. M. Oppeneer and J. A. Mydosh, Nat. Mater. 8, 337 (2009).

[41] A. V. Balatsky, A. Chantis, H. P. Dahal, D. Parker, and J. X. Zhu, Phys. Rev. B 79, 214413 (2009).

[42] P. Chandra, P. Coleman, J. A. Mydosh, and V. Tripathi, Nature (London) 417, 831 (2002).

[43] J. G. Rau and H-Y. Kee, Phys. Rev. B 85, 245112 (2012).

[44] S.-I. Fujimori, Y. Saitoh, T. Okane, A. Fujimori, H. Yamagami, Y. Haga, E. Yamamoto, and Y. Onuki, Nat. Phys. 3, 618 (2007).

[45] G. Zwicknagl, A. N. Yaresko, and P. Fulde, Phys. Rev. B 65, 081103(R) (2002).

[46] P. Chandra, P. Coleman, and R. Flint, Nature (London) 493, 621 (2013).

[47] P. S. Riseborough, B. Coqblin, and S. G. Magalhaes, Phys. Rev. B 85, 165116 (2012).

[48] H. Harima, K. Miyake, and J. FLouquet, J. Phys. Soc. Jpn. 79, 033705 (2010).

[49] C. Pépin, M. R. Norman, S. Burdin, and A. Ferraz, Phys. Rev. Lett. 106, 106601 (2011).

[50] C. Thomas, S. Burdin, C. Pépin, and A. Ferraz, Phys. Rev. B 87, 014422 (2013).

[51] S. Burdin, C. Thomas, C. Pépin, A. Ferraz, and C. Lacroix, arXiV:1406.7141.

[52] B. Coqblin and J. R. Schrieffer, Phys. Rev. 185, 847 (1969).

[53] A. M. Sengupta and A. Georges, Phys. Rev. B 52, 10295 (1995).

[54] J. R. Iglesias, C. Lacroix, and B. Coqblin, Phys. Rev. B 56, 11820 (1997).

[55] A. A. Abrikosov, Zh. Eksp. Teor. Fiz. 48, 990 (1965) [JETP 21, 66 (1965)].

[56] C. Lacroix and M. Cyrot, Phys. Rev. B 20, 1969 (1979).

[57] P. Coleman, Phys. Rev. B 28, 5255 (1983).

[58] N. Read, D. M. Newns, and S. Doniach, Phys. Rev. B 30, 3841 (1984).

[59] J. Kondo, Prog. Theor. Phys. 32, 37 (1964).

[60] P. Fazekas and P. W. Anderson, Philos. Mag. 30, 423 (1974).

[61] G. Baskaran, Z. Zou, and P. W. Anderson, Solid State Commun. 63, 973 (1987).

[62] W. H. Press, B. P. Flannery, S. A. Teukolsky, and W. T. Vetterling, Numerical Recipes (Cambridge University Press, Cambridge, UK, 1989).

[63] S. Burdin, A. Georges, and D. R. Grempel, Phys. Rev. Lett. 85, 1048 (2000).

[64] S. Burdin, D. R. Grempel, and A. Georges, Phys. Rev. B 66, 045111 (2002).

[65] B. H. Bernhard, B. Coqblin, and C. Lacroix, Phys. Rev. B 83, 214427 (2011).

[66] S. Burdin, M. Grilli, and D. R. Grempel, Phys. Rev. B 67, 121104(R) (2003).

[67] T. Senthil, S. Sachdev, and M. Vojta, Phys. Rev. Lett. 90, 216403 (2003).

[68] A. Hackl and M. Vojta, Phys. Rev. B 77, 134439 (2008).

[69] I. Paul, C. Pépin, and M. R. Norman, Phys. Rev. Lett. 98, 026402 (2007).

[70] C. Pépin, Phys. Rev. Lett. 98, 206401 (2007).

[71] I. Paul, C. Pépin, and M. R. Norman, Phys. Rev. B 78, 035109 (2008).

[72] R. Okazaki, T. Shibauchi, H. J. Shi, Y. Haga, T. D. Matsuda, E. Yamamoto, Y. Onuki, H. Ikeda, and Y. Matsuda, Science 331, 439 (2011). 
[73] S. Tonegawa, K. Hashimoto, K. Ikada, Y.-H. Lin, H. Shishido, Y. Haga, T. D. Matsuda, E. Yamamoto, Y. Onuki, H. Ikeda, Y. Matsuda, and T. Shibauchi, Phys. Rev. Lett. 109, 036401 (2012).

[74] M. A. Ruderman and C. Kittel, Phys. Rev. 96, 99 (1954).

[75] T. Kasuya, Prog. Theor. Phys. 16, 45 (1956).

[76] K. Yoshida, Phys. Rev. 106, 893 (1957).

[77] L. D. Landau, Zh. Eksp. Teor. Fiz. 30, 1058 (1956) [Sov. Phys.JETP 3, 920 (1957)].

[78] D. Pines and P. Noziéres, Theory of Quantum Liquids (Benjamin, New York, 1966), Vol. I.

[79] P. Noziéres, J. Low Temp. Phys. 17, 31 (1974).

[80] J. M. Luttinger, Phys. Rev. 119, 1153 (1960).

[81] J. M. Luttinger and J. C. Ward, Phys. Rev. 118, 1417 (1960).

[82] A. A. Abrikosov, L. P. Gorkov, and I. E. Dzyaloshinski, Methods of Quantum Field Theory in Statistical Physics (Dover, New York, 1963).

[83] P. Coleman, J. B. Marston, and A. J. Schofield, Phys. Rev. B 72, 245111 (2005).

[84] L. Isaev and I. Vekhter, Phys. Rev. Lett. 110, 026403 (2013).

[85] J. S. Hall and T. Timusk, Philos. Mag. 10, 1 (2014).

[86] J. Schoenes, C. Schonenberger, J. J. M. Franse, and A. A. Menovsky, Phys. Rev. B 35, 5375 (1987).

[87] Y. S. Oh, Kee Hoon Kim, P. A. Sharma, N. Harrison, H. Amitsuka, and J. A. Mydosh, Phys. Rev. Lett. 98, 016401 (2007).

[88] S. Chatterjee, J. Trinckauf, T. Hänke, D. E. Shai, J. W. Harter, T. J. Williams, G. M. Luke, K. M. Shen, and J. Geck, Phys. Rev. Lett. 110, 186401 (2013).
[89] R. Yoshida, Y. Nakamura, M. Fukui, Y. Haga, E. Yamamoto, Y. Onuki, M. Okawa, S. Shin, M. Hirai, Y. Muraoka, and T. Yokoya, Phys. Rev. B 82, 205108 (2010).

[90] F. L. Boariu, C. Bareille, H. Schwab, A. Nuber, P. Lejay, T. Durakiewicz, F. Reinert, and A. F. Santander-Syro, Phys. Rev. Lett. 110, 156404 (2013).

[91] K. Matsuda, Y. Kohori, T. Kohara, K. Kuwahara, and H. Amitsuka, Phys. Rev. Lett. 87, 087203 (2001).

[92] S.-H. Baek, M. J. Graf, A. V. Balatsky, E. D. Bauer, J. C. Cooley, J. L. Smith, and N. J. Curro, Phys. Rev. B 81, 132404 (2010).

[93] M. Yokoyama, H. Amitsuka, K. Tenya, K. Watanabe, S. Kawarazaki, H. Yoshizawa, and J. A. Mydosh, Phys. Rev. B 72, 214419 (2005).

[94] H. Amitsuka, K. Matsuda, I. Kawasaki, K. Tenya, M. Yokoyama, C. Sekine, N. Tateiwa, T. C. Kobayashi, S. Kawarazaki, and H. Yoshizawa, J. Magn. Magn. Mater. 310, 214 (2007).

[95] S. Burdin, Proceedings of the NATO Advanced Research Workshop on Properties and Application of Thermoelectric Materials, Hvar, Croatia 2008, edited by Veljko Zlatić and Alex C. Hewson (Springer, Dordrecht, 2009).

[96] J. R. Jeffries, N. P. Butch, B. T. Yukich, and M. B. Maple, Phys. Rev. Lett. 99, 217207 (2007).

[97] E. Hassinger, G. Knebel, T. D. Matsuda, D. Aoki, V. Taufour, and J. Flouquet, Phys. Rev. Lett. 105, 216409 (2010).

[98] K. Hasselbach, J. R. Kirtley, and P. Lejay, Phys. Rev. B 46, 5826 (1992). 